\title{
Fine-Grained Multi-label Sexism Classification Using a Semi-Supervised Multi-level Neural Approach
}

\author{
Harika Abburi $^{1}$ D $\cdot$ Pulkit Parikh $^{1} \cdot$ Niyati Chhaya ${ }^{2}$. Vasudeva Varma ${ }^{1}$
}

Received: 20 March 2021 / Revised: 26 June 2021 / Accepted: 31 July 2021 / Published online: 17 August 2021

(c) The Author(s) 2021

\begin{abstract}
Sexism, a permeate form of oppression, causes profound suffering through various manifestations. Given the increasing number of experiences of sexism shared online, categorizing these recollections automatically can support the battle against sexism, since it can promote successful evaluations by gender studies researchers and government representatives engaged in policy making. In this paper, we examine the fine-grained, multi-label classification of accounts (reports) of sexism. To the best of our knowledge, we consider substantially more categories of sexism than any related prior work through our 23-class problem formulation. Moreover, we present the first semi-supervised work for the multi-label classification of accounts describing any type(s) of sexism. We devise self-training-based techniques tailor-made for the multi-label nature of the problem to utilize unlabeled samples for augmenting the labeled set. We identify high textual diversity with respect to the existing labeled set as a desirable quality for candidate unlabeled instances and develop methods for incorporating it into our approach. We also explore ways of infusing class imbalance alleviation for multi-label classification into our semisupervised learning, independently and in conjunction with the method involving diversity. In addition to data augmentation methods, we develop a neural model which combines biLSTM and attention with a domain-adapted BERT model in an endto-end trainable manner. Further, we formulate a multi-level training approach in which models are sequentially trained using categories of sexism of different levels of granularity. Moreover, we devise a loss function that exploits any label confidence scores associated with the data. Several proposed methods outperform various baselines on a recently released dataset for multi-label sexism categorization across several standard metrics.
\end{abstract}

Keywords Sexism classification · Semi-supervised learning · Multi-label classification · Fine-grained categorization · Multi-level

\section{Introduction}

Sexism, defined as stereotyping, prejudice, or discrimination based on a person's sex, occurs in various overt and subtle forms, permeating personal as well as professional spaces.

Harika Abburi

harika.a@ research.iiit.ac.in

Pulkit Parikh

pulkit.parikh@ research.iiit.ac.in

Niyati Chhaya

nchhaya@adobe.com

Vasudeva Varma

vv@iiit.ac.in

1 IIIT-Hyderabad, Hyderabad, India

2 Adobe Research, Bangalore, India
While men and boys are also harmed by sexism, women and girls suffer the brunt of sexist mindsets and resultant wrongdoings. With increasingly many people sharing recollections of sexism experienced or witnessed by them, the automatic classification of these accounts into well-conceived categories of sexism can help fight this oppression, as it can better equip authorities formulating policies and researchers of gender studies to analyze sexism.

The detection of sexism differs from and can complement the classification of sexism. In a forum where instances of sexism are mixed with other posts unrelated to sexism, sexism detection can be used to identify the posts on which to perform sexism classification. Moreover, we observe the distinction between sexist statements (e.g., posts whereby one perpetrates sexism) and the accounts of sexism suffered or witnessed (e.g., personal recollections shared as part of the \#metoo movement). We also note the prior work 
on detecting or classifying personal stories of sexual harassment and/or assault $[10,21]$. In this paper, we focus on classifying an account (report) of sexism involving any set of categories of sexism.

Most of the existing research on sexism classification [4, 19,20 ] considers at most five categories of sexism. Further, the majority of prior approaches associate only one category of sexism with an instance of sexism. Having mutually exclusive categories of sexism is unreasonable and limiting, as substantiated by the given example. 'A colleague once saw me washing my coffee mug before leaving the office and "joked" if I was practicing for my "home duties" It's sad that he doesn't see the problem with men not bearing half the load of household work'. Associated categories for this account of sexism are 'Role stereotyping': False generalizations about some roles being more suitable for women; also applies to similar mistaken notions about men, 'Moral policing': The promotion of discriminatory guidelines for women under the pretense of morality; also applies to statements that feed into such narratives and 'Hostile work environment': Sexism suffered at the workplace; also applies when sexism perpetrated by a colleague elsewhere makes working worrisome for the victim.

To the best of our knowledge, Parikh et al. [28] is the only work that explores the multi-label categorization of accounts of sexism using machine learning and considers more than three categories of sexism. It provides the largest dataset containing accounts drawn from 'Everyday Sexism Project' ${ }^{1}$, where experiences of sexism are shared from all over the world. The textual accounts are annotated using 23 categories of sexism formulated with the help of a social scientist. However, they perform sexism classification among 14 categories derived by merging some sets of categories. This prohibits distinguishing within category pairs such as \{moral policing, victim blaming\} and \{motherhood-related discrimination, menstruation-related discrimination $\}$. We overcome this limitation by carrying out a fine-grained (23class) classification by building on the same labeled dataset.

Most existing approaches for the categorization of sexism are entirely supervised in nature (use no unlabeled data). The biggest labeled dataset available for sexism classification [28] comprises around 13, 000 accounts. In contrast, the accounts of sexism narrated on just the 'Everyday Sexism Project' website comfortably number in several hundred thousands. Effectively tapping this large volume of data has the potential to enhance the classification performance by overcoming potential weaknesses stemming from the limited training data, especially because of the fine-grained aspect of the problem. As far as we are aware, the only existing approach that uses unlabeled data for sexism classification

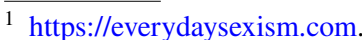

does so for merely fine-tuning a pre-trained model used for computing sentence representations [28]. We formulate the first set of methods for utilizing unlabeled instances in a more involved manner for sexism classification. The proposed data augmentation techniques are broadly based on self-training, a semi-supervised learning paradigm, in terms of the workflow. We augment the existing labeled data through the selective addition of pseudo-multi-labeled unlabeled samples.

We develop our semi-supervised methods keeping in mind the fact that, unlike in single-label multi-class (or binary) classification, a single instance can be tagged with up to 23 categories in our multi-label multi-class classification. We identify textual diversity with reference to (a subset of) the original labeled set as a useful constituent in the evaluation of candidate unlabeled instances and construct mechanisms of building it into our approach. We also seek to incorporate the desirable quality of low class imbalance into it in ways suited for multi-label semi-supervised classification. We present multiple procedures for combining these elements into a unified semi-supervised learning approach. We also add the unlabeled instances that are similar to the original labeled set that are hard to classify. We generate label confidence scores associated with each sample for all the pseudo-labeled samples that are selected through these semi-supervised data augmentation methods. As the probability predicted by the classifier will have some critical information about each label, we calculate the label confidence scores associated with each label in each sample. We formulate the loss function which utilizes these label confidence scores as a weights.

We also proposed neural architecture for the multilabel classification of accounts of sexism, which integrates domain-adapted BERT model named as tBERT along with biLSTM attention into an end-to-end training for sexism classification. As the domain-adapted tBERT model is tuned on unlabeled accounts of sexism, it may generate better sentence representation for sexism classification than the representations produced by original BERT model. These representations are complemented by sentence representations built from word embeddings as a function of trainable neural network parameters. To produce the final representation, the concatenated sentence representation is then passed to biLSTM followed by attention.

As there is a scarcity of labeled data for few categories in 23 categories of sexism, we devise a multi-level training approach for multi-label sexism classification where we train models sequentially at different categorization levels. The problem of scarcity can be alleviated by reducing the number of categories via proper merging, but it does not serve our purpose of fine-grained classification. Hence, we use training using reduced category sets as supervised pretraining steps for the final 23-class classification to benefit 
from the higher sample to category ratios associated with these pre-training steps. We initialize (most of) the weights of the model at each level with the corresponding weights of the model trained at the previous level (with fewer categories). We integrate a proposed neural model with multi-level training and our loss function to form a method that outperforms numerous baselines with a clear margin.

Our key contributions are summarized below.

- To the best of our knowledge, this is the first work to consider as many as 23 categories for sexism classification. We introduce a set of semi-supervised methods to augment the labeled data that are tailored made for multilabel multi-class sexism classification.

- We devise mechanisms aimed at enhancing the textual diversity in the resultant expanded labeled set, alleviating the skew in the original class distribution and favoring samples which are hard to classify.

- We propose a neural architecture combining biLSTM and an attention mechanism with a domain-tailored BERT model that allows for end-to-end training for multi-label sexism classification.

- We propose a loss function that makes use of the label confidence scores associated with the each pseudolabeled sample in augmented data.

- We develop a multi-level training method for multi-label sexism classification wherein we train models sequentially at different levels.

- Several proposed methods outperform numerous baselines, including the existing state-of-the-art, across various established metrics.

The rest of our paper is structured as follows. Section 2 describes related prior work. Section 3 discusses the semisupervised data augmentation methods that we propose for multi-label sexism classification. Section 4 describes the proposed multi-label sexism classification approaches and also the loss function. Experimental results and observations are provided in Sect. 5. We conclude with a summary in Sect. 6.

\section{Related Work}

In this section, we review the work on the classification of sexism after noting some distantly related work. Though our work involves classifying accounts of sexism, prior work on the classification of sexist or misogynous statements (e.g., tweets wherein one perpetrates sexism or misogyny) is also included in this review. We also present work relating to the identification and classification of hate speech, as some of it applies to our work to a certain degree in the detection of sexist hate.
Melville et al. [25] apply topic modeling to data gathered from the 'Everyday Sexism Project' and map the semantic relations between the topics. ElSherief et al. [16] study user involvement with posts related to gender-based violence and their language variations. Warner and Hirschberg [39] detect hate speech using an SVM classifier using brown clusters, $\mathrm{n}$-grams and the occurrence of words. Corazza et al. [11] explore different datasets from different languages to examine multilingual hate speech detection. The authors used models such as Bi-LSTM and SVM to construct models for detecting hate speech. We note that sexism detection can complement sexism classification by preceding it to remove posts unrelated to sexism. The detection of sexism is performed by some hate speech classification approaches that include sexism as a category of hate [12]. [5] explored various deep learning approaches such as fastText, RNN and $\mathrm{CNN}$ to classify the given tweet as racist, sexist and neither. Gao et al. [18] perform hate speech detection in a weakly supervised fashion. Waseem and Hovy [40] classified tweets as sexist, racist or neither using character n-grams along with extra-linguistic features. Zhang and Luo [46] explored the word embeddings with a combination of GRU and CNN and skipped CNN to classify tweets as sexism, racism, both and non-hate. Qian et al. [33] provide a hierarchical conditional variational autoencoder model for fine-grained hate speech classification. Rodríguez-Sánchez et al. [34] detect the sexism on tweets using BILSTM and BERT models in Spanish language. Chiril et al. [9] introduced the method for detecting sexism reports/denunciations from actual sexist material that is specifically addressed to a target or defines a target. [8] created an dataset for sexism detection in French tweets and explore several deep learning architectures like BERT, CNN, CNN-LSTM, bi-LSTM with attention. Frenda et al. [17] present an approach for detecting sexism and misogyny from tweets. Plaza-Del-Arco et al. [32] perform hate speech detection in Spanish tweets for the partially superposed domains of xenophobia and misogyny by comparing different machine learning and deep learning approaches.

Burnap and Williams [6] build a data-driven model of cyberhate to identify disability, race and sexual orientation using bag-of-words, dictionary and text parser to extract typed dependencies. Schrading et al. [35] identify pieces of text discussing domestic abuse on Reddit. Nobata et al. [27] extract linguistic, character $\mathrm{n}$-grams, semantic and syntactic features to detect abusive comments and analyze the abusive language over time from the corpus of Yahoo Finance and News comments. Van Hee et al. [37] use n-gram and sentiment lexicon features to identify and classify cyberbullying. Agrawal and Awekar [3] explore cyberbullying detection across social media platforms using deep learning methods including biLSTM with attention. Unlike these papers, we seek to categorize sexism and misogyny, which include hate speech directed at women but are not limited to hate. Zhong 
et al. [47] detect cyberbullying in comments posted on Instagram images through features obtained from captions and images.

Karlekar and Bansal [21] investigate RNN, CNN and a combination of $\mathrm{RNN}$ and $\mathrm{CNN}$ for categorizing personal experiences of sexual harassment into one or more of three classes. For the classification of personal stories of sexual harassment, [42] uses a density matrix encoder inspired by quantum mechanics. Khatua et al. [22] explore deep learning methods to classify sexual violence into one of four categories. In Anzovino et al. [4], tweets identified as misogynist are classified as discredit, stereotype and objectification, dominance, or derailing, sexual harassment, and threats of violence using features involving n-grams, part of speech (POS) tags and text embedding. A four-class categorization of sexist tweets is carried out by Jafarpour et al. [19] which deal with threats and harassment by improving the training data using knowledge graphs like ConceptNet. Suvarna and Bhalla [36] identified victim blaming language on Twitter using transfer learning-based classification method. Chowdhury et al. [10] detect personal recollections of sexual harassment from Twitter posts. In Jha and Mamidi [20], tweets are classified as hostile, benevolent or non-sexist using biLSTM with attention, fastText and SVM. While its categorization of sexism relates to how it is stated, our work concentrates on aspects such as where it occurs, who perpetrates it and what an instance of sexism involves.

Parikh et al. [28] address the multi-label categorization of accounts of sexism. They create the largest dataset and provide state-of-the-art classifier for the sexism classification. The classifier combines sentence embeddings generated using a BERT [13] model with those generated from ELMo [31] and GloVe [30] embeddings using biLSTM with attention and CNN. Abburi et al. [1] explore a multitask approach for semi-supervised sexism classification that deploys three auxiliary tasks such as estimating the topic proportion distribution, predicting the cluster label and detecting an account of sexism without inflicting any manual labeling cost. They also explore the objective functions that make use of label correlations present in the training data. As far as we know, our work presents the first semi-supervised data augmentation approaches for the multi-label classification of accounts describing any type(s) of sexism.

\section{Semi-Supervised Data Augmentation for Multi-label Sexism Classification}

This section presents proposed methods that employ semisupervised learning for classifying an account of sexism (also referred to as a post henceforth) such that the categories can co-occur. We begin by laying the groundwork for the description of our methods.

\subsection{Basic Self-Training}

Self-training [2, 44] is a semi-supervised learning approach that helps augment the set of labeled instances by selectively adding unlabeled samples. For performing a task such as classification (or regression) in the presence of unlabeled and labeled data, a typical self-training method first trains a model (e.g., classifier) on the labeled instances. Next, it applies the model to the unlabeled instances and identifies a subset of them to be added to the training set, along with the predicted labels, based on criteria such as the confidence scores associated with the model's predictions. After expanding the training set by adding this pseudo-labeled subset, a new classifier is trained on the augmented set. This process is repeated until some stopping criteria such as the stabilization of model parameters and the number of iterations completed are satisfied.

\subsection{Base Model}

We employ a deep learning model that generates the output probabilities for all the labels through the sigmoid nonlinearity on its last (dense) layer as our base classifier. The loss function used for training the model is a weighted mean of label-wise binary cross-entropy values.

\subsection{Proposed Approach}

While there exists some prior work on the classification of textual records involving sexism, most methods are supervised. We observe that the accounts of sexism reported on the 'Everyday Sexism Project' website alone hugely outnumber those provided in the biggest existing labeled dataset for sexism classification, Parikh et al. [28]. The performance of a sexism classification method can be improved by leveraging this sizable chunk of unlabeled data. We explore semisupervised techniques based on self-training to utilize unlabeled accounts of sexism. We devise multiple methods of expanding the set of labeled data using unlabeled instances befitting the multi-label nature of instances of sexism.

We first formulate a basic method based on self-training tailor-made for the multi-label problem configuration. We also propose other methods built on top of it with a view to (1) improving the proportions of positive (relevant) samples across categories, (2) improving the class balance keeping in mind the mutual non-exclusivity of category labels, (3) encouraging textual diversity in the newly labeled (pseudolabeled) data relative to the original training set and (4) favoring the samples which are hard to classify. We also develop some combinations involving these proposed methods. The augmented data generated by any of our methods can be used for training any supervised classification model. 
We now describe the proposed semi-supervised methods in depth.

\subsubsection{Basic Self-Training for Multi-label Classification}

The most fundamental, indispensable factor in determining which unlabeled instances should be considered to be added to the original labeled set during self-training is the confidence of correctness associated with each prediction. In single-label (multi-class) classification, one can simply treat the classification probability corresponding to the one predicted class as this confidence. This procedure is inapplicable to a multi-label case wherein the base classifier outputs a probability of applicability for each label. We observe that our baseline multi-label classifier generates the probabilities following sigmoid (as opposed to softmax) nonlinearities and that predictions need to be made by rounding the perlabel (per-sample) probabilities $p_{j}$. Since this implies that the probability linked with the prediction for unlabeled sample $u_{k} \in U$ for label $l_{j}$ is either $\hat{p}_{k j}^{\sigma}$ or $1-\hat{p}_{k j}^{\sigma}$, we mandate that at least one of these two quantities exceeds a threshold (hyper-parameter $T$ ) for each label for $u_{k}$ to qualify for being added to the labeled set.

\subsubsection{Improving Positive Sample Proportions Across Categories (IPSPC)}

In addition to the basic confidence-based check, this method subjects unlabeled instances to another qualifying test relating to the number of predicted labels using the base classifier. The intuition is that the higher the number of predicted labels, the greater the number of labels for which relevant (positive) samples are contributed. Since the number of positive samples is outweighed by the negative counterpart by a substantial margin across most labels, we attempt to counter this skew by picking the unlabeled instances with a certain minimum number of predicted labels (hyper-parameter $\left.P_{\min }\right)$. In order to maximize the label correctness of the chosen pseudo-labeled set, we also avoid candidate samples with an unreasonably high number of predicted labels (hyper-parameter $P_{\max }$ ).

\subsubsection{Favoring Low-Support Labels}

While the previous method seeks to improve the per-category ratios of positive to negative sample counts generally, this method attempts to correct the class imbalance between categories while creating the augmented dataset. We present two methods that order unlabeled samples (adhering to the checks proposed earlier) based on notions of support that we design for multi-label classification. We then pick the lowest-support $T o p_{p}$ percent of the samples as the pseudo-labeled set in each iteration, where hyper-parameter $T o p_{p}$ is empirically determined. For the first method (Support.uniform), support for an unlabeled sample $u_{k} \in U$ is defined as

$\operatorname{support\_ uniform}\left(u_{k}\right)=\frac{\sum_{j \in P_{k}+} \sum_{i=1}^{M^{*}} y_{i j}}{\left|P_{k}^{+}\right|}$,

where $P_{k}{ }^{+}$is the set of labels predicted for $u_{k}, U$ denotes the unlabeled data, $M^{*}$ is the number of labeled samples in the current iteration, and $y_{i j}$ is 1 if category $l_{j}$ is given for sample $x_{i}$ in the labeled data and 0 otherwise.

Since this method considers the average coverage in the labeled data across all predicted labels for a sample, a sample linked with some weak labels (labels with low frequencies in the labeled data) and some extremely strong labels may get rated lower than one linked with no weak label and some moderately strong labels. In Support.weakest, we explicitly take into account only the weak labels for the notion of support. In each iteration, we determine weak labels based on the coverage (frequency) of each label in the current labeled data. Specifically, a label $l_{j}$ is weak if $\sum_{i=1}^{M^{*}} y_{i j}<S_{m}$ and strong otherwise, where $S_{m}$ is a hyperparameter. We disregard all strong labels while calculating the support for an unlabeled sample with at least one weak predicted label. For samples with predictions involving no weak classes, we resort to the previous support computation. For the rest, we compute the support as follows.

$$
\operatorname{support\_ weakest}\left(u_{k}\right)=\frac{\sum_{j \in P_{k}{ }^{+}} v_{j}}{\left|\left\{z \mid \sum_{i=1}^{M^{*}} y_{i z}<S_{m}, z \in P_{k}{ }^{+}\right\}\right|} \text {, }
$$

where $v_{j}=\sum_{i=1}^{M^{*}} y_{i j}$ if $\sum_{i=1}^{M^{*}} y_{i j}<S_{m}$ and 0 otherwise.

\subsubsection{Seeking Textual Diversity}

We identify the utility of selecting pseudo-labeled data such that it complements the existing labeled data as opposed to being an expanded version of it, especially in terms of linguistic characteristics. This family of methods aims to introduce greater textual diversity with respect to (a subset of) the current labeled data in the set of samples of being added in each iteration. Candidate unlabeled samples (meeting the qualifying criteria proposed in the first two methods) are ranked as per how far they are from existing labeled samples in terms of the corresponding vector representations. We create a variant of the state-of-the-art deep learning model for the original labeled dataset given in Parikh et al. [28] for generating the embedding for the text of a given sample. Cosine distance is used as the distance metric. The highestrank $T o p_{p}$ percent of the samples are added to the labeled data in each iteration. In Diversity.uniform, diversity for a sample $u_{k} \in U$ is given by, 
diversity_uniform $\left(u_{k}\right)=\frac{\sum_{i=1}^{M^{*}} \cos \_d i s t\left(p o s t \_r e p\left(u_{k}\right), p o s t \_r e p\left(x_{i}\right)\right)}{M^{*}}$,

where post_rep refers to the vector representation for a sample. Diversity.uniform picks the most distinct unlabeled samples w.r.t. the current labeled set in a label-independent manner. We develop the Diversity.label method to incorporate per label diversity, avoiding indiscriminate comparisons against all labeled samples. For a candidate sample, for each label predicted for it, we compute the average of the distances against only the labeled samples bearing that label. Each candidate is scored using the average of these label-wise averages. Our formulation can be expressed as,

diversity_label $\left(u_{k}\right)=\frac{\sum_{j \in P_{k}{ }^{+}} \frac{\sum_{i=1}^{M^{*}} y_{i j} \cos \_d i s t\left(p o s t \_r e p\left(u_{k}\right), p o s t \_r e p\left(x_{i}\right)\right)}{\sum_{i=1}^{M^{*}} y_{i j}}}{\left|P_{k}^{+}\right|}$

\subsubsection{Combining Previously Proposed Methods}

We develop two ways of integrating our methods favoring low-support labels and seeking greater textual diversity to explore if their individual strengths combine well.

(1) Score computation: We calculate the combined score for a candidate $u_{k}$ from unlabeled data $U$ as follows.

$\operatorname{score}\left(u_{k}\right)=\frac{\text { diversity_uniform }\left(u_{k}\right)\left(\text { or diversity_label }\left(u_{k}\right)\right)}{\text { support_uniform }\left(u_{k}\right)\left(\text { or support_weakest }\left(u_{k}\right)\right)}$

The or in the equation above simply indicates that we consider all four (2X2) combinations stemming from the previous two proposed families of methods. From the pseudolabeled candidate instances which pass the screenings previously described, Top $p$ percent of the instances with the highest combined scores are chosen for labeled data augmentation.

(2) Intersection: In each iteration, we employ a method favoring low-support labels and a method seeking greater textual diversity each to pick the pseudo-labeled samples. We then intersect the sets of pseudo-labeled samples selected by these two methods and augment the current labeled set with the resultant set of samples. In this way of combining the previously proposed methods also, we explore pairing each of the two methods favoring low-support labels with each of the two methods seeking greater textual diversity.

\subsubsection{Favoring Hard Samples}

In this family of methods, we augment the labeled dataset with pseudo-labeled samples that are similar to labeled samples that we deem hard to classify. We define the notion of hard samples in a label-specific manner as well as generically. Each method orders unlabeled samples (that adhere to the qualifying criteria proposed in the first two methods) based on their vector representation similarities with (a subset of) the labeled samples identified as hard based on the corresponding notion of hardness. Then, we choose the most similar $T_{o p}$ percent of the samples as the pseudo-labeled set in each iteration, where $T_{o p}$ is a hyper-parameter.

We hold out a part of the training data and train a new classifier (following the same architecture as the base model) on the rest. We identify the hard samples from this held-out data using the new classifier's predictions on it. For each sample in the held-out data, for each label, we compute the probability of the correct binary (applicable or not) prediction from the classifier-produced probabilities. For sample $x_{h}$ from the held-out data and label $l_{j}$, this probability $\hat{p}_{h j}^{c}$ is calculated in the following manner.

$\hat{p}_{h j}^{c}=\left\{\begin{array}{l}\hat{p}_{h j}^{\sigma}, \text { if } y_{h j}=1 \\ 1-\hat{p}_{h j}^{\sigma}, \text { otherwise }\end{array}\right.$

In a proposed method which we name hard.uniform, we deem a held-out data sample $x_{h}$ hard if the mean of the probabilities $\hat{p}_{h j}^{c}$ across all labels is less than a threshold (hyperparameter $T_{h u}$ ). We also experiment with replacing the threshold-based check for picking the hard labeled samples; we sort all held-out data samples $x_{h}$ by the means of the probabilities $\hat{p}_{h j}^{c}$ across all labels and deem a certain number of top samples hard. The threshold-based method outperforms this variant. Once the hard labeled samples are chosen, we compute the average similarity of for an unlabeled sample $u_{k}$ with them using cosine similarity as follows.

hard_uniform_similarity $\left(u_{k}\right)$

$=\frac{\sum_{h=1}^{H^{*}} \text { cos_similarity }\left(\text { post_rep }\left(u_{k}\right), \text { post_rep }\left(x_{h}\right)\right)}{H^{*}}$,

where $H^{*}$ is total number of hard labeled samples identified and post_rep refers to the vector representation for a sample computed using the method mentioned in 3.3.4.

In our hard.label method, we identify hard labeled samples from the held-out data per label. Sample $x_{h}$ is identified as hard for label $l_{j}$ if $\hat{p}_{h j}^{c}$ is below a threshold (hyper-parameter $\left.T_{h l}\right)$. We experiment with a variant aimed at removing the threshold-based check in this case too; for each label $l_{j}$, we sort all held-out data samples $x_{h}$ by the probabilities $\hat{p}_{h j}^{c}$ and deem a certain number of top samples hard. This variant 
under-performs the threshold-based method. We make use of the label-wise sets of hard samples as follows. For an unlabeled sample $u_{k}$, for each label predicted for it with a non-empty hard sample set, we compute the average of the cosine similarities to the hard samples for that label. The final similarity score is the average of these label-wise averages. In the event that the hard sample sets for all the labels predicted for $u_{k}$ are empty, it is assigned hard_uniform_similarity $\left(u_{k}\right)$.

hard_label_similarity $\left(u_{k}\right)$

$$
=\frac{\sum_{j \in\left\{j \mid H_{j}^{*}>0, j \in P_{k}+\right\}} \frac{\sum_{h=1}^{H_{j}^{*}} \text { cos_similarity }\left(\text { post_rep }\left(u_{k}\right), \text { post__eep }\left(x_{h}\right)\right)}{H_{j}^{*}}}{\left|\left\{j \mid H_{j}^{*}>0, j \in P_{k}^{+}\right\}\right|},
$$

where $H_{j}^{*}$ is the number of hard labeled samples identified for label $l_{j}$.

\subsubsection{Generating Label Confidence Scores}

For each unlabeled sample $u_{k}$ that is added to the training data by any of the data augmentation methods, a confidence score is computed. Confident score $c_{k j}$ for each unlabeled sample, per each predicted probability $\hat{p}_{k j}^{\sigma}$ is computed as follows,

$c_{k j}= \begin{cases}\hat{p}_{k j}^{\sigma}, & \text { if } \hat{p}_{k j}^{\sigma} \geq 0.5, \forall j \\ 1-\hat{p}_{k j}^{\sigma}, & \text { otherwise }\end{cases}$

These confidence scores can be used during the fully supervised classification.

\section{Proposed Multi-Label Sexism Classification Approaches}

In this section, we detail our approaches for carrying out the multi-label classification of accounts of sexism. We begin the section with the description of the proposed neural architecture. Next, we discuss the multi-level training approach that we develop for multi-label classification by creating organizing the categories of sexism in a meaningful hierarchy. We conclude the section by specifying the details of the proposed loss function utilizing label confidence scores.

\subsection{Proposed Sexism Classification Architecture}

Figure 1 shows our proposed sexism classification architecture. Each account of sexism (raw input text) is represented as multiple 3-d tensors, each of which embeds each word of each sentence. The ELMo and GloVe word embedding methods generate $\mathcal{R}^{|S| \times|W| \times d_{e}}$ and $\mathcal{R}^{|S| \times|W| \times d_{g}}$ tensors, respectively, where $|S|$ denotes the maximum number sentences per post (account of sexism), $|W|$ is the maximum number of words per sentence, and $d_{e}$ and $d_{g}$ represent the embedding dimensions for ELMo and GloVe, respectively. We also employ a domain-adapted BERT variant named tBERT for embedding words; it produces a $\mathcal{R}^{|S| \times|W| \times d_{b}}$ tensor, where $d_{b}$ is the tBERT embedding dimension. tBERT is created by further training a pre-trained, generic BERT model in an unsupervised manner using unlabelled accounts of sexism with a view to producing more effective representations for sexism classification than those produced by off-the-shelf BERT models. We incorporate tBERT into our end-to-end training for sexism classification (the weights of tBERT are updated during the training).

We construct vector representations for the word-embedded sentences in the three 3-D tensors using bidirectional LSTM and an associated attention mechanism [43]. For each sentence, the biLSTM layer produces $|W| h$-dimensional hidden states (one for each of the $|W|$ time steps). These hidden states are aggregated into a vector representation by the attention layer (for each sentence). Overall, this results in three $\mathcal{R}^{|S| \times h}$ tensors (one corresponding to each of the three 3 -D word embedding tensors), where $h$ is the bi-LSTM output length. These sentences representing 2-D tensors are concatenated to produce a $\mathcal{R}^{|S| \times 3 h}$ tensor. The sequence of sentence vectors in this tensor is then passed to biLSTM followed by the attention, resulting in the post representation. Finally, a fully connected layer with the sigmoid nonlinearity generates the output probabilities for all the labels.

As the cross-entropy loss used for single-label multiclass classification problem is not applicable to the multilabel classification setup, we employ the extended binary cross-entropy (EBCE) loss used in Parikh et al. [28]. It is formulated as a weighted mean of label-wise binary crossentropy values, where the weights are meant for neutralizing the class imbalance.

\subsection{Multi-level Training Using a Category Hierarchy}

We devise a coarse-to-fine training approach for multi-label sexism classification wherein we sequentially train models using categories of sexism of different levels of granularity. The motivation behind this coarse-to-fine training is the scarcity of labeled data for some categories in the 23-class fine-grained categorization scheme. The scarcity problem can be alleviated by simply merging categories appropriately, but it does not serve the fine-grained classification objective. Hence, we use the training on a reduced category set (created through category merging) as a supervised pre-training step. The intuition is that the higher sample to category ratio could lead to better weights and initializing the final model with (most of) those weights (as opposed to randomly) could therefore yield a superior fine-grained classifier. Moreover, this supervised pre-training may also in 


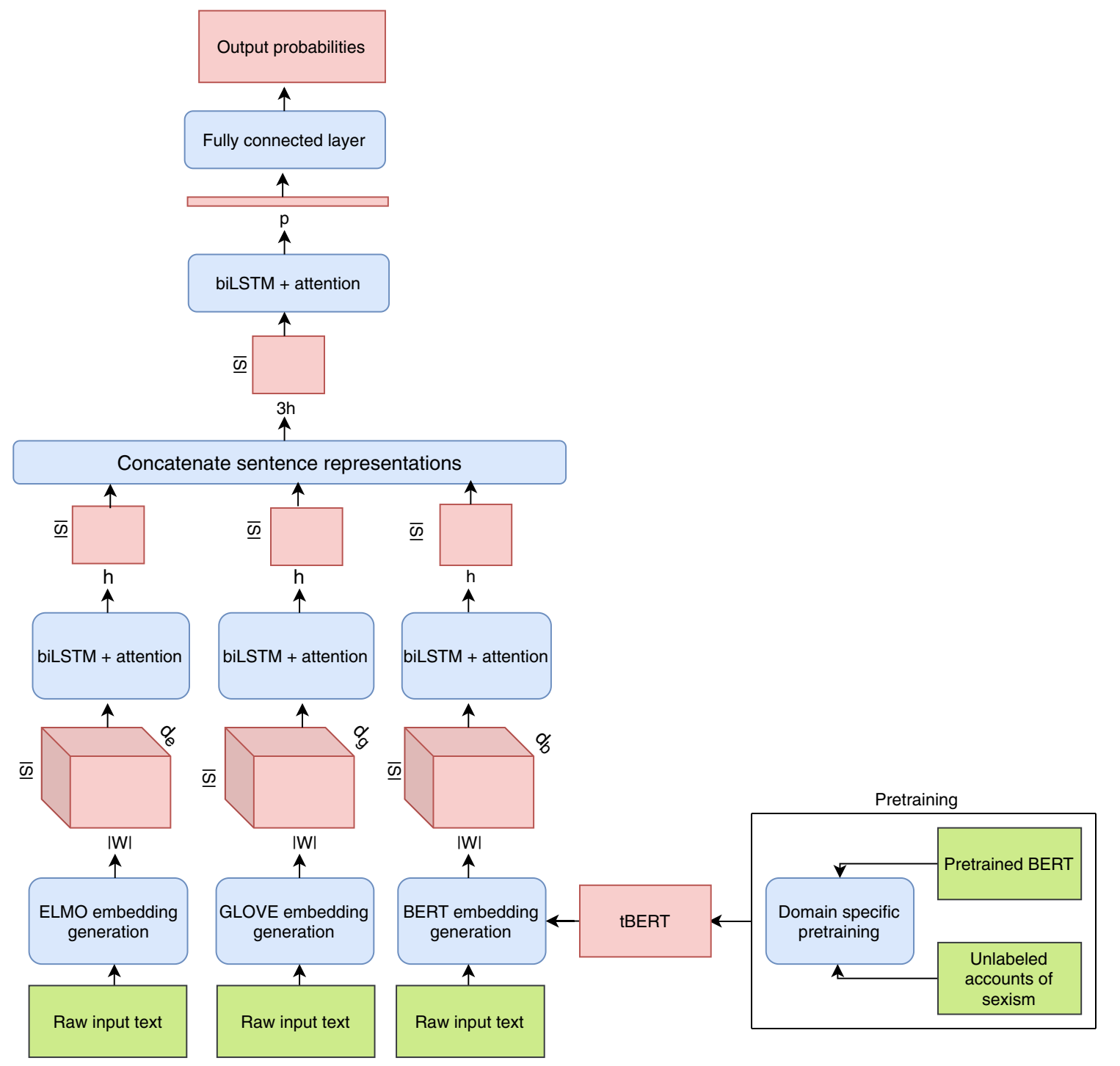

Fig. 1 Proposed Sexism Classification Architecture

turn benefit from another instance of supervised pre-training involving even fewer categories. Therefore, we carry out a sequence of training steps at different categorization levels. The architectures of the models at all levels are the same except for the final, dense layer. At each level, the dense layer of each model has the same number of output units as the category set size.

In order to perform the multi-level training, we create a category hierarchy from the original 23 fine-grained categories of sexism under the direction of a social scientist. Figure 2 specifies this three-level hierarchy of categories of sexism. $m()$ denotes the merging of categories. At each level, the merged categories are shown in a different color. The category hierarchy consists of 8 categories at the most abstract level (level 1) and 15 categories in the middle level.
First, we train a model using the level 1 categories with a final, dense (fully connected) layer with 8 output units. The values of the weights of this trained level 1 model except those of the final, dense layer are used to initialize the corresponding weights in the level 2 model. We then train this model using the training data modified using the 15 middlelevel categories. The same is repeated for the third, final, fine-grained level. Except for the final layer, the level 3 model's weights are initialized with the values of the weights of the trained level 2 model. We then train the 23-category level 3 model, which we perform the desired fine-grained sexism classification with. 


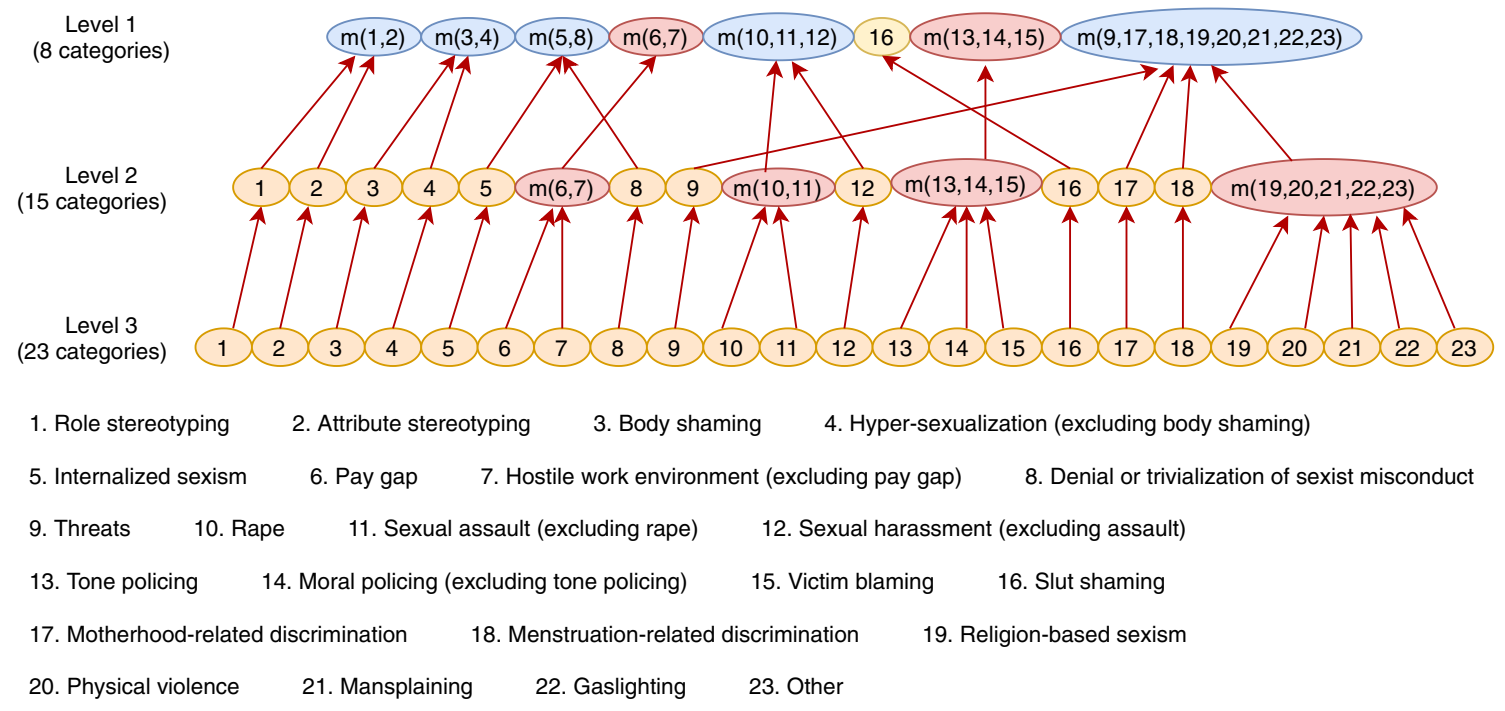

Fig. 2 Category hierarchy for multi-level training for multi-label sexism classification

\subsection{Proposed Confidence-Modified Binary Cross-Entropy Loss}

We formulate a variant of the extended binary cross-entropy (EBCE) loss that is aimed at utilizing the label confidence scores. These per-label confidence scores may be generated by a pseudo-labeling data augmentation method. The proposed loss, which we refer to as Confidence-modified Binary Cross-Entropy (CBCE), also involves weights through which we see to correct the class imbalance. We formulate the CBCE loss as follows.

$C B C E=-\frac{1}{L} \sum_{i=1}^{L} \frac{1}{N} \sum_{j=1}^{N} w_{j y_{i j}} c_{i j}\left\{y_{i j} \log \left(\hat{p}_{i j}^{\sigma}\right)+\left(1-y_{i j}\right) \log \left(1-\hat{p}_{i j}^{\sigma}\right)\right\}$

Here, $L$ is the number of samples and $N$ is the number of classes. $\hat{p}_{i j}^{\sigma}$ is the predicted probability of label $l_{j}$ being applicable to sample $x_{i} \cdot c_{i j}$ is the confidence score for label $l_{j}$ for sample $x_{i} \cdot y_{i j}$ is defined as follows.

$y_{i j}=\left\{\begin{array}{l}1, \text { if label } l_{j} \text { is applicable to sample } x_{i} \\ 0, \text { otherwise }\end{array}\right.$

The weights for correcting class imbalance $w_{j z}$ are computed as follows.

$w_{j z}=\frac{L}{2\left|\left\{x_{i} \mid y_{i j}=z, 1 \leq i \leq L\right\}\right|}$

\section{Experiments}

This section discusses all the experimental results of the proposed methods compared to several baseline methods and analyzes related to our method. Our code and all the hyperparameter values used are available at this link. ${ }^{2}$

\subsection{Dataset}

Parikh et al. [28] introduce a dataset comprising 13, 023 accounts of sexism, each account of sexism labeled with at least one of the 23 categories. They formulated the 23 categories of sexism under the direction of a social scientist by considering gender-related discourse and campaigns [14, $15,24,26]$ as well as possible impact on public policy. Most of the 10 annotators involved had studied topics related to gender and/or sexuality formally. Moreover, a three-phase method of annotation was pursued to ensure that the categorization of each account of sexism in the final dataset involved the labeling of it by at least two annotators. Finally, after the three phases of annotation, the dataset comprises 13,023 accounts of sexism, where each account of sexism is labeled with at least one of the 23 categories. Table 1 provides the description of the categories. We use this original labeled dataset (only) to train all supervised baselines.

We provide a linguistic analysis of this labeled data using Linguistic Inquiry and Word Count (LIWC), a text analysis tool [29]. We focus on the LIWC scores for the Work, Money, Religion and Body categories. Details concerning

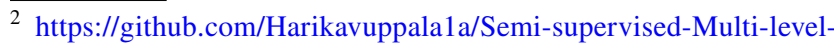
neural-approach.
} 
Table 1 Descriptions of the categories of sexism used in the dataset [28]

\begin{tabular}{|c|c|}
\hline Category & Description \\
\hline Role stereotyping & $\begin{array}{l}\text { Socially constructed false generalizations about certain roles being more appropriate for } \\
\text { women; also applies to such misconceptions about men }\end{array}$ \\
\hline Attribute stereotyping & $\begin{array}{l}\text { Mistaken linkage of women with some physical, psychological, or behavioral qualities or likes/ } \\
\text { dislikes; also applies to such false notions about men }\end{array}$ \\
\hline Body shaming & $\begin{array}{l}\text { Objectionable comments or behavior concerning appearance including the promotion of certain } \\
\text { body types or standards }\end{array}$ \\
\hline Hyper-sexualization (excluding body shaming) & Unwarranted focus on physical aspects or sexual acts \\
\hline Internalized sexism & The perpetration of sexism by women via comments or other actions \\
\hline Pay gap & Unequal salaries for men and women for the same work profile \\
\hline Hostile work environment (excluding pay gap) & $\begin{array}{l}\text { Sexism encountered by an employee at the workplace; also applies when a sexist misdeed com- } \\
\text { mitted outside the workplace by a co-worker makes working uncomfortable for the victim }\end{array}$ \\
\hline Denial or trivialization of sexist misconduct & Denial or downplaying of sexist wrongdoings \\
\hline Threats & $\begin{array}{l}\text { All threats including wishing for violence or joking about it, stalking, threatening gestures, or } \\
\text { rape threats }\end{array}$ \\
\hline Rape & FBI's expanded definition of rape \\
\hline Sexual assault (excluding rape) & Any sexual contact without consent; unwanted touching \\
\hline Sexual harassment (excluding assault) & Any sexually objectionable behavior \\
\hline Tone policing & Comments or actions that cause or aggravate restrictions on how women communicate \\
\hline Moral policing (excluding tone policing) & $\begin{array}{l}\text { The promotion of discriminatory codes of conduct for women in the guise of morality; also } \\
\text { applies to statements that feed into such codes and narratives }\end{array}$ \\
\hline Victim blaming & $\begin{array}{l}\text { The act of holding the victim responsible (fully or partially) for sexual harassment, violence, or } \\
\text { other sexism perpetrated against her }\end{array}$ \\
\hline Slut shaming & $\begin{array}{l}\text { Inappropriate comments made about women 1) deviating from conservative expectations relat- } \\
\text { ing to sex or 2) dressing in a certain way when it gets linked to sexual availability }\end{array}$ \\
\hline Motherhood-related discrimination & $\begin{array}{l}\text { Shaming, prejudices, or other discrimination or misconduct related to the notion of motherhood; } \\
\text { also applies to the violation of reproductive rights }\end{array}$ \\
\hline Menstruation-related discrimination & Shaming, prejudices, or other discrimination or wrongdoings related to periods \\
\hline Religion-based sexism & Sexist discrimination or prejudices stemming from religious scriptures or constructs \\
\hline Physical violence (excluding sexual violence) & $\begin{array}{l}\text { Domestic abuse, murder, kidnapping, confinement, or other physical acts of violence linked to } \\
\text { sexism }\end{array}$ \\
\hline Mansplaining & $\begin{array}{l}\text { A woman being condescendingly talked down to by a man; also applies when a man gives an } \\
\text { unsolicited advice or explanation to a woman related to something she knows well that she } \\
\text { disapproves of }\end{array}$ \\
\hline Gaslighting & Sexist manipulation of the victim through psychological means into doubting her own sanity \\
\hline Other & Any type of sexism not covered by the above categories \\
\hline
\end{tabular}

how the LIWC scores are computed can be found in the LIWC 2015 [29]. Table 2 shows the scores for these LIWC categories for all the categories of sexism in the dataset. For each class of sexism, we compute the LIWC scores for all posts tagged with that class label and take the mean of all those scores to obtain the category-level scores reported. We observe the highest scores for the Work and Money LIWC categories for Pay gap. As expected, the maximum score for the Religion LIWC category is found for Religion-based sexism. For the Body LIWC category, we find the highest score for Body shaming. Table 2 also lists 4 -grams from the textual accounts of sexism associated with each category of sexism.
In this paper, we also devise semi-supervised methods to automatically expand this dataset. Unlabelled instances of sexism are obtained from 'Everyday Sexism Project' which has several hundred thousand accounts of sexism from observers and survivors. We shortlist 70, 000 shortest unlabeled instances of sexism containing a minimum of 7 words each. Short instances are selected in order to maximize the similitude to the labeled data [28]. Our data augmentation methods select a subset of these 70,000 accounts of sexism for augmenting the training data.

\subsection{Evaluation Metrics}

Evaluation metrics for multi-label classification problems differ from the standard metrics used in cases where the 
Table 2 Linguistic analysis of the labeled dataset

\begin{tabular}{|c|c|c|c|c|c|c|}
\hline ID & Category of sexism & LIWC Work & LIWC Money & LIWC Religion & LIWC Body & 4-grams \\
\hline 1 & Role stereotyping & 5.466 & 1.059 & 0.110 & 0.374 & $\begin{array}{l}\text { 'man of the house', 'to be a nurse', 'to } \\
\text { cook and clean' }\end{array}$ \\
\hline 2 & Attribute stereotyping & 3.543 & 0.895 & 0.071 & 0.739 & $\begin{array}{l}\text { 'drive like a girl', 'funny for a girl', } \\
\text { 'pink is for girls' }\end{array}$ \\
\hline 3 & Body shaming & 1.765 & 0.188 & 0.085 & 3.322 & $\begin{array}{l}\text { 'im too ugly to', 'if you lost weight', } \\
\text { 'me a fat bitch' }\end{array}$ \\
\hline 4 & $\begin{array}{l}\text { Hyper-sexualization (excluding body } \\
\text { shaming) }\end{array}$ & 3.102 & 0.484 & 0.071 & 2.589 & $\begin{array}{l}\text { 'the tits on that', 'looking me up and', } \\
\text { 'a piece of meat' }\end{array}$ \\
\hline 5 & Internalized sexism & 3.079 & 0.453 & 0.078 & 1.168 & $\begin{array}{l}\text { 'told by my mother', 'she suggested } \\
\text { that my', 'told by my female' }\end{array}$ \\
\hline 6 & Pay gap & 10.081 & 3.806 & 0.128 & 0.203 & $\begin{array}{l}\text { 'being paid more than', 'the exact same } \\
\text { job', 'several thousand more than' }\end{array}$ \\
\hline 7 & $\begin{array}{l}\text { Hostile work environment (excluding } \\
\text { pay gap) }\end{array}$ & 8.070 & 0.764 & 0.063 & 1.042 & $\begin{array}{l}\text { 'first day working at', 'the only female } \\
\text { in', 'my boss called me' }\end{array}$ \\
\hline 8 & $\begin{array}{l}\text { Denial or trivialization of sexist } \\
\text { misconduct }\end{array}$ & 2.514 & 0.309 & 0.091 & 1.135 & $\begin{array}{l}\text { 'i should be flattered', 'said it was just', } \\
\text { 'a bit of fun' }\end{array}$ \\
\hline 9 & Threats & 1.647 & 0.401 & 0.041 & 0.948 & $\begin{array}{l}\text { 'followed by a man', 'he would rape } \\
\text { me', 'to follow me home' }\end{array}$ \\
\hline 10 & Rape & 2.019 & 0.208 & 0.102 & 0.823 & $\begin{array}{l}\text { 'on top of me', 'raped at a party', 'been } \\
\text { raped twice and' }\end{array}$ \\
\hline 11 & Sexual assault (excluding rape) & 2.023 & 0.318 & 0.043 & 3.251 & $\begin{array}{l}\text { 'his crotch against my', 'i was groped } \\
\text { by', 'i felt a hand' }\end{array}$ \\
\hline 12 & Sexual harassment (excluding assault) & 2.378 & 0.413 & 0.039 & 1.763 & $\begin{array}{l}\text { 'whistled at while walking', 'looking } \\
\text { me up and', 'asking me to come' }\end{array}$ \\
\hline 13 & Tone policing & 2.771 & 0.208 & 0.000 & 0.887 & $\begin{array}{l}\text { 'me not to swear', 'that it was unlady- } \\
\text { like', 'tell me not to' }\end{array}$ \\
\hline 14 & $\begin{array}{l}\text { Moral policing (excluding tone polic- } \\
\text { ing) }\end{array}$ & 3.566 & 0.501 & 0.164 & 0.988 & $\begin{array}{l}\text { 'back to the kitchen', 'should be at } \\
\text { home', 'should go out with' }\end{array}$ \\
\hline 15 & victim blaming & 1.767 & 0.126 & 0.076 & 1.916 & $\begin{array}{l}\text { 'was asking for it', 'fault for wearing } \\
\text { tight', 'it was her fault' }\end{array}$ \\
\hline 16 & Slut shaming & 1.977 & 0.575 & 0.042 & 1.479 & $\begin{array}{l}\text { 'shouted whore at me', 'how much i } \\
\text { cost', 'called a slag by' }\end{array}$ \\
\hline 17 & Motherhood-related discrimination & 5.095 & 0.576 & 0.189 & 0.604 & $\begin{array}{l}\text { 'planning on having children', 'get mar- } \\
\text { ried and have', 'are you a pregnant' }\end{array}$ \\
\hline 18 & Menstruation-related discrimination & 2.634 & 0.213 & 0.074 & 0.795 & $\begin{array}{l}\text { 'time of the month', 'you on your } \\
\text { period', 'must be on her' }\end{array}$ \\
\hline 19 & Religion-based sexism & 3.173 & 0.525 & 4.675 & 0.848 & $\begin{array}{l}\text { 'my religion only males', 'that in my } \\
\text { religion', 'piece of clothing that' }\end{array}$ \\
\hline 20 & $\begin{array}{l}\text { Physical violence (excluding sexual } \\
\text { violence) }\end{array}$ & 1.156 & 0.233 & 0.094 & 2.198 & $\begin{array}{l}\text { 'spat in my face', 'pushed me into a', } \\
\text { 'murdered by her husband' }\end{array}$ \\
\hline 21 & Mansplaining & 3.585 & 0.599 & 0.018 & 0.234 & $\begin{array}{l}\text { 'cut off by a', 'to mansplain to me', 'he } \\
\text { was embarrassing me' }\end{array}$ \\
\hline 22 & Gaslighting & 0.000 & 0.453 & 0.220 & 0.800 & $\begin{array}{l}\text { 'boyfriend would guilt me', 'guilt me } \\
\text { into agreeing', 'agreeing to various } \\
\text { sexual' }\end{array}$ \\
\hline 23 & Other & 3.846 & 0.568 & 0.079 & 0.669 & $\begin{array}{l}\text { 'to me as mrs', 'being referred to as', } \\
\text { 'calling me young lady' }\end{array}$ \\
\hline
\end{tabular}

Bold values indicate the best performance

classes cannot co-occur. We report results for a number of established metrics, namely Subset Accuracy $(S A)$, instancebased F1 $\left(F_{\text {ins }}\right)$, instance-based accuracy $(A c c), \mathrm{F} 1$ macro
$\left(F_{\text {mac }}\right)$ and F1 micro $\left(F_{\text {mic }}\right)[28,45]$. Subset Accuracy, which measures the fraction of the exact matches, is the strictest metric. 
These metrics are mathematically expressed as follows.

$$
\begin{gathered}
F_{\text {inst }}=\frac{2 P_{\text {inst }} R_{\text {inst }}}{P_{\text {inst }}+R_{\text {inst }}}, \text { where } \\
P_{\text {ins }}=\frac{1}{P} \sum_{i=1}^{P} \frac{\left|\mathbf{y}_{\mathbf{i}} \cap \hat{\mathbf{y}}_{i}\right|}{\left|\hat{\mathbf{y}}_{i}\right|}, \\
R_{\text {ins }}=\frac{1}{P} \sum_{i=1}^{P} \frac{\left|\mathbf{y}_{\mathbf{i}} \cap \hat{\mathbf{y}}_{i}\right|}{\left|\mathbf{y}_{\mathbf{i}}\right|} \\
F_{\text {mac }}=\frac{1}{Q} \sum_{j=1}^{Q} F\left(T P_{j}, F P_{j}, F N_{j}\right), \\
F_{\text {mic }}=F\left(\sum_{j=1}^{Q} T P_{j}, \sum_{j=1}^{Q} F P_{j}, \sum_{j=1}^{Q} F N_{j}\right),
\end{gathered}
$$

$$
\begin{aligned}
\text { whereTP }_{j} & =\left|\left\{x_{i} \mid l_{j} \in\left(\mathbf{y}_{\mathbf{i}} \cap \hat{\mathbf{y}}_{i}\right), 1 \leq i \leq P\right\}\right|, \\
F P_{j} & =\left|\left\{x_{i} \mid l_{j} \in\left(\hat{\mathbf{y}}_{i}-\mathbf{y}_{\mathbf{i}}\right), 1 \leq i \leq P\right\}\right| \\
F N_{j} & =\left|\left\{x_{i} \mid l_{j} \in\left(\mathbf{y}_{\mathbf{i}}-\hat{\mathbf{y}}_{i}\right), 1 \leq i \leq P\right\}\right|,
\end{aligned}
$$

$F\left(T P^{*}, F P^{*}, F N^{*}\right)=\frac{2 T P^{*}}{2 T P^{*}+F N^{*}+F P^{*}}$

$A c c=\frac{1}{P} \sum_{i=1}^{P} \frac{\left|\mathbf{y}_{\mathbf{i}} \cap \hat{\mathbf{y}}_{i}\right|}{\left|\mathbf{y}_{\mathbf{i}} \cup \hat{\mathbf{y}}_{i}\right|}$

$S A=\frac{1}{P} \sum_{i=1}^{P} 1_{\mathbf{y}_{\mathbf{i}}=\hat{\mathbf{y}}_{i}}$

Here, $P$ denotes number of posts. $\mathbf{y}_{\mathbf{i}}$ is set of true labels applicable to post $x_{i} . \hat{\mathbf{y}}_{i}$ is the set of predicted labels for post $x_{i}$. $Q$ denotes the number of classes and $l_{j}$ denotes the $j^{t h}$ of the $\mathrm{Q}$ labels.

\subsection{Baselines}

All the below-mentioned deep learning architectures end with a dense layer with the sigmoid activation and trained with the EBCE loss.

\section{Random}

For each test sample, labels are selected randomly as per their normalized frequencies in the training data.

\section{Traditional Machine Learning (TML)}

We experiment with logistic regression (LR), support vector machine (SVM) and random forests (RF) classifiers. All the classifiers applied on two feature sets, namely TFIDF on word unigrams and bigrams (Word-ngrams) and the average of the ELMo vectors [31]. This gives rise to six combinations: word-ngrams with LR, word-ngrams with
SVM, word-ngrams with RF, ELMO with LR, ELMO with SVM and ELMO with RF.

Deep Learning (DL:) LSTM-based Architectures

- biLSTM: The word embeddings corresponding to each post are fed through a bidirectional LSTM.

- biLSTM-Attention and Hierarchical-biLSTM-Attention: The biLSTM-Attention is similar to biLSTM, but with the attention scheme from Yang et al. [43]. Hierarchical-biLSTM-Attention is similar to Yang et al. [43], but GRUs are replaced with LSTMs. For each post, the word embeddings are passed through the biLSTM-attention to create a sentence representation. The sentence representation is then fed to another biLSTM-attention.

- USE-biLSTM-Attention and BERT-biLSTM-Attention : Sentence embeddings are generated using USE [7] and BERT via bert-as-service [41] separately and passed through a biLSTM with attention.

\section{CNN-biLSTM and CNN-based Architectures}

- C-biLSTM: This architecture is somewhat similar to approach [21] and one of the variants of C-LSTM architecture [48]. After applying the convolution operation on each post's word vectors, the feature maps are stacked with the filter dimension in order to generate a series of window vectors, which are then passed through biLSTM.

- CNN-biLSTM-Attention: This architecture is similar to Wang et al. [38], where word embeddings of each sentence are fed to convolutional and max-over-time pooling layers. These sentence representations are then fed to a biLSTM with attention.

- CNN-Kim: Word vectors of a post are passed through convolutional and max-over-time pooling layers similar to $\operatorname{Kim}[23]$.

\section{Semi-supervised: Classification methods}

- tBERT-biLSTM-Attention: This architecture is similar to BERT-biLSTM-Attention except that the pre-trained BERT model is fine-tuned using unlabeled instances of sexism [28].

- Opti-DL: This is the best-performing model by [28]. The neural model concatenates sentence representations obtained using a BERT [13] model tuned using unlabeled instances of sexism with those generated from ELMo [31] and GloVe [30] embeddings separately using biLSTM with an attention scheme. The combined sentence vectors are passed through biLSTM with attention to produce the post representation. 
Table 3 Results for traditional machine learning baselines

\begin{tabular}{llllll}
\hline Approach & $F_{\text {ins }}$ & $F_{\text {mac }}$ & $F_{\text {mic }}$ & Acc & SA \\
\hline Word-ngrams-LR & 0.544 & 0.188 & 0.492 & 0.454 & $\mathbf{0 . 2 8 7}$ \\
Word-ngrams-SVM & 0.453 & 0.227 & 0.413 & 0.331 & 0.107 \\
Word-ngrams-RF & 0.538 & 0.246 & 0.482 & 0.444 & 0.272 \\
ELMO-LR & $\mathbf{0 . 5 7 6}$ & $\mathbf{0 . 2 6 1}$ & $\mathbf{0 . 5 3 5}$ & $\mathbf{0 . 4 7 5}$ & 0.279 \\
ELMO-SVM & 0.546 & $\mathbf{0 . 2 6 1}$ & 0.501 & 0.431 & 0.206 \\
ELMO-RF & 0.374 & 0.100 & 0.330 & 0.307 & 0.185 \\
\hline
\end{tabular}

Bold values indicate the best performance

\section{Data augmentation methods}

- Random Sampling + Opti-DL: We randomly choose the same number of unlabelled samples as those generated by our best data augmentation method (Diversity.label $\cap$ Support.weakest) using proposed architecture (PA) as a base classifier, label them using the Opti-DL model and augment the original labeled set with them, and then train Opti-DL on the expanded set.

- Mean-based self-training + Opti-DL: We adapt basic self-training [44] approach for multi-label classification. Iteratively, we train Opti-DL model to generate the pseudo-label unlabeled samples and add them to the training set only if the mean of the model given probabilities for the predicted class labels exceeds certain threshold.

\subsection{Results}

For all deep learning approaches, the pre-processing steps we perform involve eliminating some non-alpha-numeric characters and additional spaces, lower casing and zero padding input tensors as necessary. Each sentence of more than 35 words is split into several sentences when splitting a post into sentences, maintaining the maximum sentence length of 35 words.

From original labeled data, $15 \%$ is used for validation, and $15 \%$ is used for testing. During the testing phase, the validation set was merged into the training set. After augmenting the original labeled data, the base classifier and the proposed architecture were also trained on the union of the augmented data and validation set for the semi-supervised methods. For all the deep learning methods, the mean of the results obtained over three runs is reported for all the metrics. For each proposed data augmentation method, data augmentation is carried out over 3 iterations.

Table 3 provides the results produced by various traditional machine learning baselines. It is observed that among all the combinations of features and classifiers experimented with the traditional machine learning, averaged ELMo embeddings with logistic regression perform best for most of the metrics.

Table 4 provides the sexism classification results for random, various deep learning and semi-supervised baselines as well as for different sets of proposed methods. The random baseline works incredibly poorly as expected, illustrating the complexity of the fine-grained multi-label classification. For the deep learning baselines, we find ELMo to be better than GloVe for word embeddings across multiple baselines and hence reported on ELMo-based results with EBCE loss. Hierarchical-biLSTM-Attention is the best deep learning baseline across all the metrics, and it outperforms its traditional machine learning counterpart. Overall the best baseline is the semi-supervised Opti-DL method. BERT model tuned on domain-specific unlabeled instances of sexism named as tBERT works better than the vanilla BERT counterpart and USE. Augmented data generated by mean-based self-training approach using Opti-DL as the base classifier performed poorly compared to Opti-DL. Randomly selecting the same number of samples from the unlabelled accounts of sexism as our best-proposed data augmentation method does and labeled them using the Opti-DL worsens its performance to a degree.

Several proposed methods outperform all baselines across all the metrics. The maximum improvement in the performance is observed for subset accuracy. For all the proposed data augmentation methods, the best classification baseline (Opti-DL) is used to generate augmented data as well as a final classifier (to predict the labels). Our best-performing data augmentation method is Diversity.label $\cap$ support. weakest, which prioritizes samples that are the most distinct compared to the existing labeled data and have the lowestsupport weak (low-coverage) predicted label sets. Among the methods that seek greater textual diversity, Diversity. uniform produces the best results for most metrics which select the samples that are more distinct in a label independent manner. Support.uniform shows the best performance among the variants of favoring low-support labels that picks the samples that has low coverage. Among the methods favoring hard samples, Hard.uniform performs best for most of the metrics. We report all the combinations involving our diversity-based and support-based methods through the computation of combined scores and intersection, where $\mathrm{S}()$ and $\cap$ denote the score-based and intersection-based integration, respectively. S(Diversity.label,Support.uniform) performs best for the score-based integration combinations.

In proposed supervised classification methods, Proposed Architecture (PA) leads to the best results where the sentence representations obtained from ELMO and GLOVE are concatenated with the tBERT (BERT tuned on unlabelled instances of sexism) representations. PA without pre-training BERT worsens its performance to a degree, as the model is not tuned on the domain-specific data. The performance of 
Table 4 Results for Deep Learning (DL) and semi-supervised baselines as well as different sets of proposed methods

\begin{tabular}{|c|c|c|c|c|c|c|}
\hline & Approach & $F_{i n s}$ & $F_{\text {mac }}$ & $F_{m i c}$ & Acc & SA \\
\hline \multirow{3}{*}{ DL baselines } & Random & 0.035 & 0.090 & 0.192 & 0.022 & 0.003 \\
\hline & biLSTM & 0.627 & 0.451 & 0.577 & 0.472 & 0.147 \\
\hline & biLSTM-Attention & 0.648 & 0.445 & 0.597 & 0.499 & 0.176 \\
\hline \multirow{10}{*}{ Semi-supervised baselines } & Hierarchical-biLSTM-Attention & 0.664 & 0.485 & 0.616 & 0.516 & 0.191 \\
\hline & USE-biLSTM-Attention & 0.566 & 0.398 & 0.525 & 0.402 & 0.061 \\
\hline & BERT-biLSTM-Attention & 0.591 & 0.397 & 0.546 & 0.431 & 0.089 \\
\hline & C-bilstm & 0.485 & 0.316 & 0.446 & 0.328 & 0.038 \\
\hline & CNN-biLSTM-Attention & 0.421 & 0.284 & 0.387 & 0.278 & 0.035 \\
\hline & CNN-Kim & 0.658 & 0.481 & 0.617 & 0.513 & 0.195 \\
\hline & tBERT-biLSTM-Attention & 0.632 & 0.435 & 0.580 & 0.472 & 0.127 \\
\hline & Opti-DL & 0.714 & 0.546 & 0.665 & 0.572 & 0.242 \\
\hline & Random Sampling + Opti-DL & 0.682 & 0.492 & 0.607 & 0.532 & 0.184 \\
\hline & Mean-based self-training +Opti-DL & 0.701 & 0.506 & 0.648 & 0.549 & 0.211 \\
\hline \multirow{16}{*}{$\begin{array}{l}\text { Opti-DL on data augmented by proposed } \\
\text { methods using Opti-DL as the base } \\
\text { classifier }\end{array}$} & Basic & 0.721 & 0.539 & 0.669 & 0.582 & 0.257 \\
\hline & IPSPC & 0.723 & 0.540 & 0.673 & 0.588 & 0.276 \\
\hline & Diversity.label & 0.726 & 0.552 & 0.679 & 0.594 & 0.288 \\
\hline & Diversity.uniform & 0.733 & 0.545 & 0.686 & 0.603 & 0.304 \\
\hline & Support.weakest & 0.726 & 0.544 & 0.678 & 0.591 & 0.279 \\
\hline & Support.uniform & 0.735 & 0.547 & 0.684 & 0.603 & 0.299 \\
\hline & Hard.label & 0.715 & 0.542 & 0.663 & 0.576 & 0.265 \\
\hline & Hard.uniform & 0.724 & 0.539 & 0.678 & 0.589 & 0.274 \\
\hline & S(Diversity.label, Support.uniform) & 0.732 & 0.544 & 0.683 & 0.601 & 0.302 \\
\hline & S(Diversity.label, Support.weakest) & 0.724 & 0.538 & 0.675 & 0.591 & 0.292 \\
\hline & S(Diversity.uniform, Support.uniform) & 0.716 & 0.551 & 0.668 & 0.578 & 0.255 \\
\hline & S(Diversity.uniform, Support.weakest) & 0.727 & 0.553 & 0.677 & 0.593 & 0.287 \\
\hline & Diversity.uniform $\cap$ Support.uniform & 0.719 & 0.553 & 0.668 & 0.583 & 0.276 \\
\hline & Diversity.uniform $\cap$ Support.weakest & 0.729 & 0.547 & 0.677 & 0.591 & 0.273 \\
\hline & Diversity.label $\cap$ Support.uniform & 0.734 & 0.551 & 0.686 & 0.606 & 0.315 \\
\hline & Diversity.label $\cap$ Support.weakest & 0.738 & 0.554 & 0.691 & 0.610 & 0.316 \\
\hline \multirow[t]{3}{*}{ Proposed classification methods } & Proposed Architecture (PA) & 0.737 & 0.566 & 0.694 & 0.607 & 0.298 \\
\hline & PA without pretraining & 0.726 & 0.557 & 0.684 & 0.596 & 0.291 \\
\hline & Multi-level PA & 0.736 & 0.565 & 0.693 & 0.609 & 0.297 \\
\hline \multirow[t]{6}{*}{ Proposed combined methods } & $\begin{array}{l}\text { PA on data augmented by Diversity.label } \cap \text { Support.weakest } \\
\text { using Opti-DL as the base classifier }\end{array}$ & 0.746 & 0.568 & 0.700 & 0.622 & 0.333 \\
\hline & $\begin{array}{l}\text { Opti-DL on data augmented by Diversity.label } \cap \text { Support. } \\
\text { weakest using PA as the base classifier }\end{array}$ & 0.736 & 0.563 & 0.688 & 0.604 & 0.299 \\
\hline & $\begin{array}{l}\text { PA on data augmented by Diversity.label } \cap \text { Support.weakest } \\
\text { using PA as the base classifier (PA-aug) }\end{array}$ & 0.750 & 0.576 & 0.706 & 0.630 & 0.341 \\
\hline & PA-aug with CBCE Loss & 0.755 & 0.581 & 0.708 & 0.635 & 0.352 \\
\hline & Multi-level PA-aug & 0.755 & 0.576 & 0.709 & 0.633 & 0.354 \\
\hline & Multi-level PA-aug with CBCE Loss & 0.757 & $\mathbf{0 . 5 8 3}$ & 0.714 & 0.638 & 0.357 \\
\hline
\end{tabular}

Bold values indicate the best performance

hierarchical multi-level training approach with PA slightly decreases compared to the PA when trained on the original labeled data. Further, we consider the best data augmentation method (Diversity.label $\cap$ support.weakest), best classification method (PA) and best baseline (Opti-DL) and tried different combinations. Out of the combined methods, PA used to generate the augmented data as well as a final classifier (PA-aug) performs best compared to the other combinations. On top of PA-aug, CBCE loss and hierarchical multi-level are experimented separately and observes that the performance is improved compared to PA-aug. Finally, the best performance is observed with a combined proposed method involving multi-level PA-aug along with our proposed CBCE loss function. 
Fig. 3 Performance of our best method (Diversity.label $\cap$ Support.weakest) and best baseline (mean-based self-training) for data augmented (using PA as the base classifier) with different neural classifiers
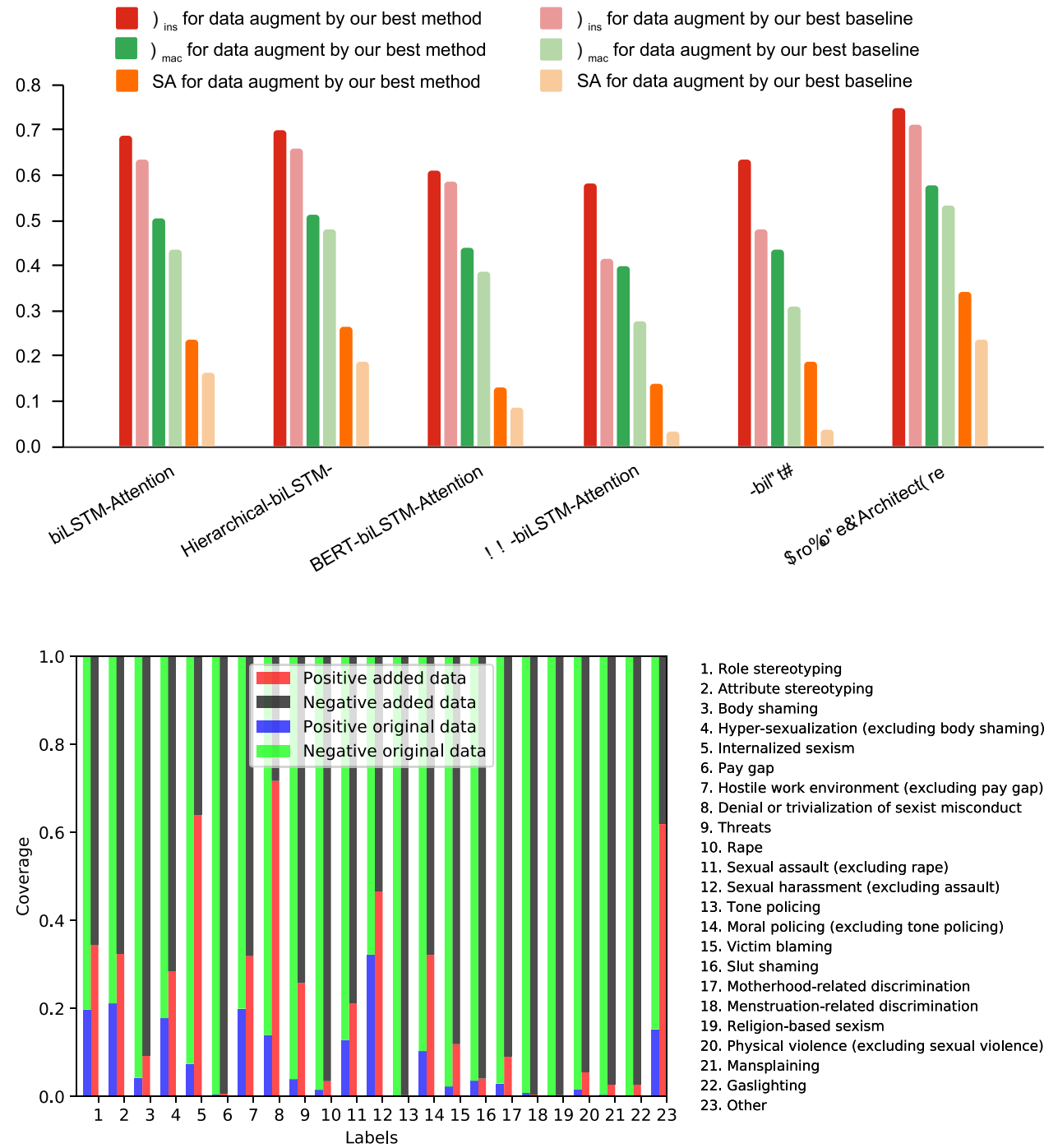
Labels

\author{
Role stereotyping \\ . Attribute stereotyping \\ 3. Body shaming \\ . Internalized sexism \\ Hostile work environment (excluding pay gap) \\ 8. Denial or trivialization of sexist misconduct \\ Threats \\ 0. Rape \\ 11. Sexual assault (excluding rape) \\ 12. Sexual harassment (excluding assault) \\ 13. Tone policing \\ 4. Moral policing (excluding tone policing) \\ 15. Victim blaming \\ 16. Slut shaming \\ 17. Motherhood-related discrimination \\ 18. Menstruation-related discrimination \\ 19. Religion-based sexism \\ 20. Physical violence (excluding sexual violence) \\ 22. Gaslighting \\ 23. Other
}

Fig. 4 Coverage of positive and negative samples per label for the original data and data added by our best method (Diversity. label $\cap$ Support.weakest) using $\mathrm{PA}$ as a base classifier
We analyze the performance of the data augmented by our best data augmentation method (Diversity.label $\cap$ Support.weakest) and best data augmentation baseline (meanbased self-training) using PA as base classifier across different neural classifiers. Figure 3 depicts the $F_{\text {ins }}, F_{\text {mac }}$ and $S A$ for five deep learning baseline classifiers and proposed architecture. The figure demonstrates the relative efficacy of our data augmented by our best augmentation method across different classifiers for all the three metrics. For CNN-based architectures, it is visible clearly that the data augment by our best augmentation method performs better with good margin compared to the data augment by best baseline.

Figure 4 highlights the coverage of positive and negative samples for each of the 23 labels in the original labeled data and contrasts it against the improved positive sample proportion in the data contributed (added data) by our best method (Diversity.label $\cap$ support.weakest) using PA as a base classifier. The ratio of the standard deviation and mean for the (positive) label coverage for the original data is 1.074, whereas its added data counterpart is 1.022, indicating that our data augmentation method also reduces the class imbalance to a degree.

Figures 5, 6, 7 portray the $F_{\text {ins }}, F_{\text {mac }}$ and $S A$ for four data augmentation methods with different combinations of PA and Opti-DL at different stages. In Fig. 5, we render the performance of our best classifier (PA) and the best classification baseline (Opti-DL) on data augmented by different data augmentation methods using Opti-DL as a base classifier. The figure shows that PA as a final classifier performed best for all the methods across all the metrics compared to OptiDL. In Fig. 6, for each data augmentation method, we depict the performance of the best classification baseline (Opti-DL) on data augmented by different methods using best classification baseline (Opti-DL) and best classifier (PA) as base classifiers. The figure demonstrates that the data generated by the best classifier (PA) perform best compared to the best classification baseline (Opti-DL) for all the data augmentation methods. In Fig. 7, for each data augmentation 
Fig. 5 Performance of our best classifier (PA) and the best classification baseline (Opti-DL) on data augmented by different methods using Opti-DL as a base classifier $\#_{\text {ins }}$ for PA on data augmented using Opti-DL $\#_{\text {mac }}$ for PA on data augmented using Opti-DL SA for PA on data augmented using Opti-DL

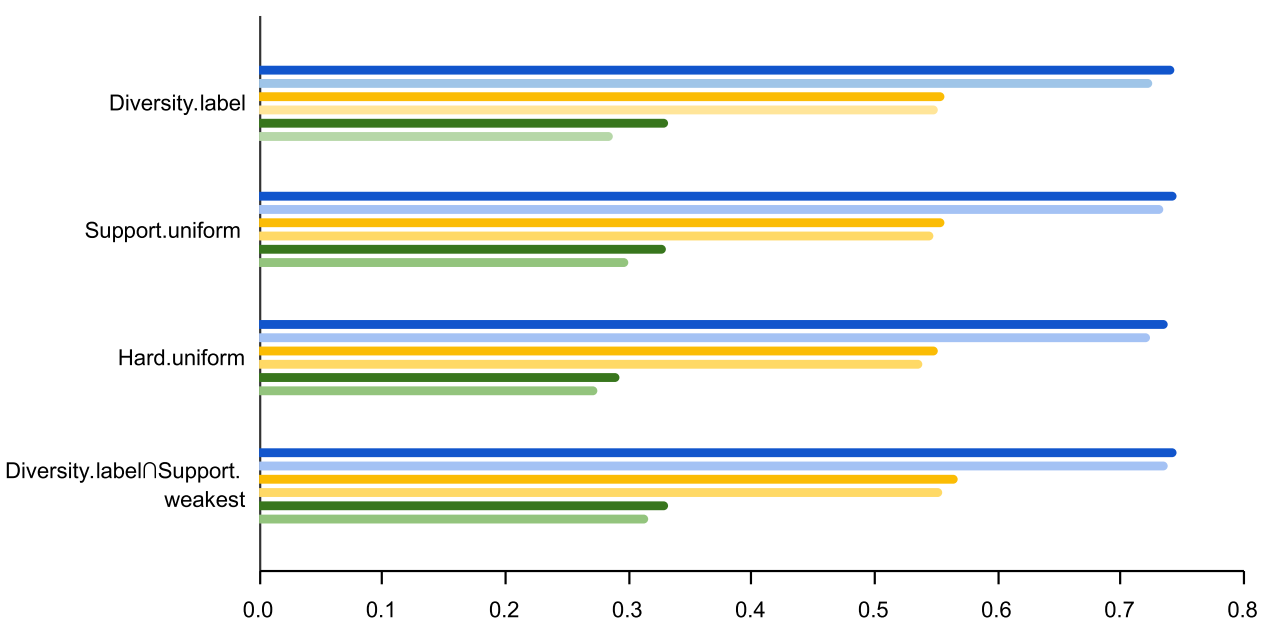

Fig. 6 Performance of the best classification baseline (OptiDL) on data augmented by different methods using Opti-DL and PA as a base classifiers
\# for Opti-DL on data augmented using PA $\#_{\text {mac }}$ for Opti-DL on data augmented using PA

SA for Opti-DL on data augmented using PA

\# for Opti-DL on data augmented using Opti-DL $\#_{\text {mac }}$ for Opti-DL on data augmented using Opti-DL SA for Opti-DL on data augmented using Opti-DL

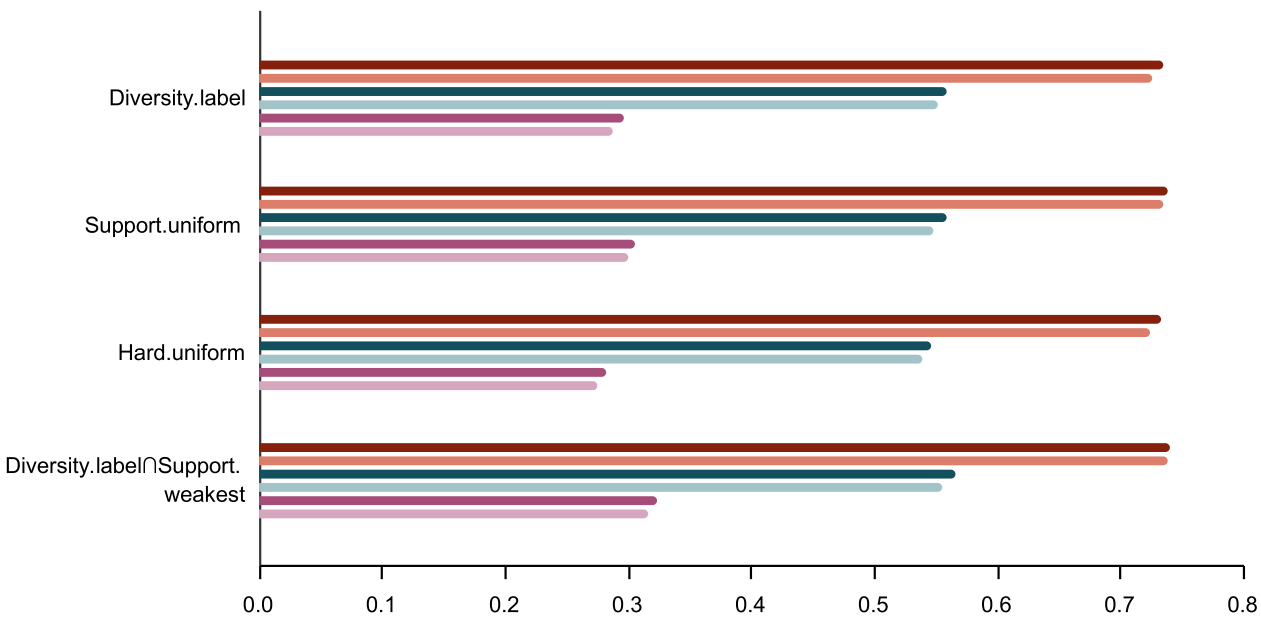

approach, we show the performance of our best classification method (PA) and the best classification baseline (Opti-DL) on data augmented by different methods using PA and Opti$\mathrm{DL}$ as base classifiers, respectively. The figure shows relative efficacy of PA at both the stages compared to the Opti-DL. Overall, Figs. 5, 6, 7 show that our PA performed well as a base classifier, as a final classifier and at both stages (as base and final classifier) for all the data augmentation methods across all the metrics compared to Opti-DL.

For the best data augmentation method (Diversity.label $\cap$ support.weakest), we also showed the effect of using our best classification method (PA) as the base classifier and/or as the final classifier. Table 5 shows a comparison of PA and the best classification baseline (Opti-DL) at different stages for all the metrics. It is observed that PA generates best augmented data as well as performed well as a final classifier compared to Opti-DL at both the stages.

Figure 8 compares the class-wise performance of our overall best method (multi-level PA-aug with CBCE loss) with that of the best baseline (Opti-DL). For each class, average of the F scores over three runs is shown for both the methods. For a majority of the classes, the F score of the proposed method outperforms the baseline F score.

Table 6 shows accounts of sexism from the test set for which our best proposed method (multi-level PA-aug with CBCE loss) makes all correct predictions, but the best baseline (Opti-DL) does not. It also provides the average cosine similarity scores w.r.t. the original labeled data and the new 
Fig. 7 Performance of our best classifier (PA) and the best classification baseline (Opti-DL) on data augmented by different methods using PA and Opti-DL as a base classifiers

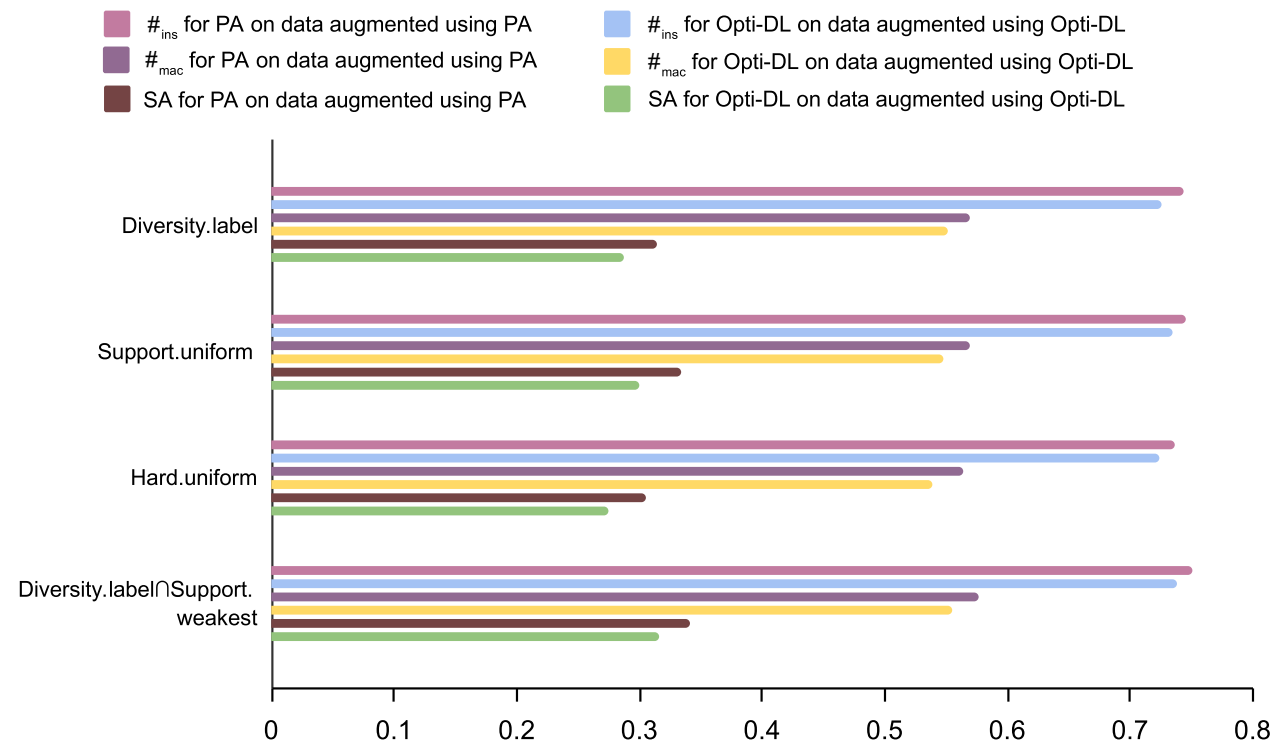

Table 5 Effect of using our best classification method (PA) as the base classifier for data augmentation method and/or as the final classifier (using Diversity.label $\cap$ support.weakest for data augmentation)

\begin{tabular}{lllll}
\hline Approach & $F_{\text {ins }}$ & $F_{\text {mac }}$ & $F_{\text {mic }}$ & Acc \\
\hline Opti-DL on data augmented using Opti-DL & 0.738 & 0.554 & 0.691 & 0.610 \\
PA on data augmented using Opti-DL & 0.746 & 0.568 & 0.700 & 0.316 \\
Opti-DL on data augmented using PA & 0.740 & 0.565 & 0.693 & 0.333 \\
PA on data augmented using PA & $\mathbf{0 . 7 5 0}$ & $\mathbf{0 . 5 7 6}$ & $\mathbf{0 . 7 0 6}$ & 0.613 \\
\hline
\end{tabular}

Bold values indicate the best performance

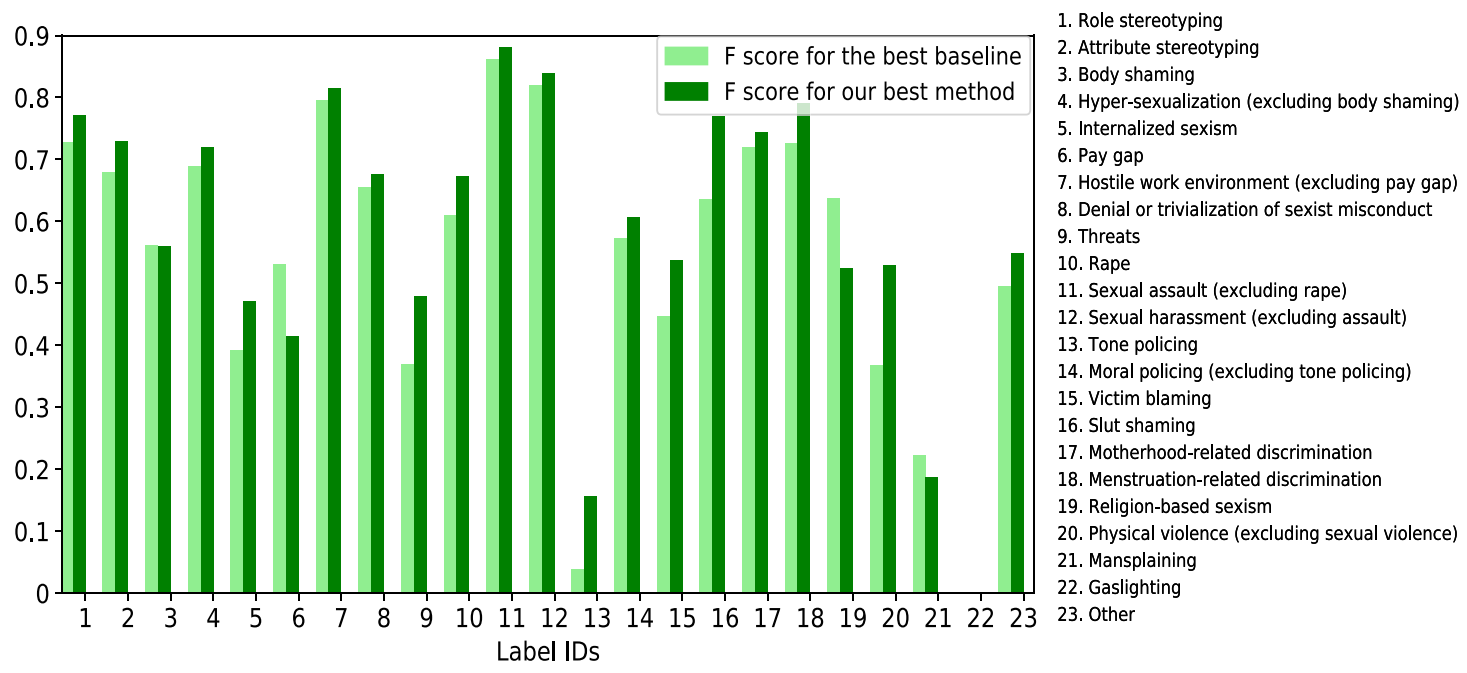

Fig. 8 Class-wise sexism classification F-scores for the best baseline (Opti-DL) and our overall best method (multi-level PA-aug with CBCE loss)

pseudo-labeled data produced by our best data augmentation method (Diversity.label $\cap$ support.weakest), computed using vector representations of the posts given by the PA. It shows that the test samples are more similar to the newly added pseudo-labeled data. We also report the per-label coverage, defined as the fraction of samples bearing the label, for the 


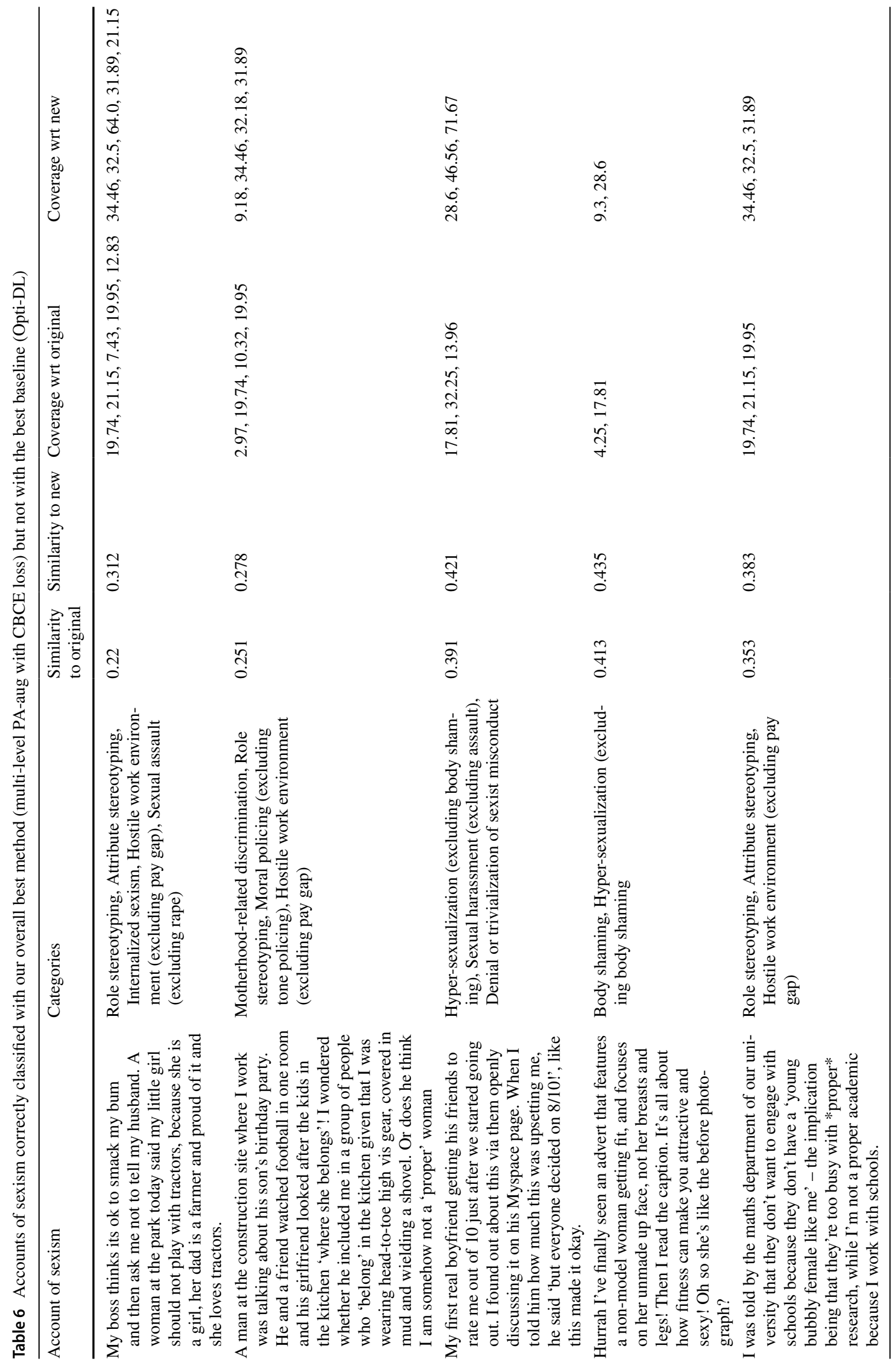


Table 7 Examples of the pseudo-labeled accounts of sexism added by our Diversity.label $\cap$ Support.weakest using PA as the base classifier

\begin{tabular}{|c|c|}
\hline Account of sexism & Categories \\
\hline $\begin{array}{l}\text { Meeting with a senior manager at work who I have met only a couple } \\
\text { of times and refers to me as voluptuous. Mentioned it to a colleague } \\
\text { to be told 'oh that's just what he's like' }\end{array}$ & $\begin{array}{l}\text { Hyper-sexualization (excluding body shaming), Hostile work environ- } \\
\text { ment (excluding pay gap), Denial or trivialization of sexist miscon- } \\
\text { duct, Sexual harassment (excluding assault)' }\end{array}$ \\
\hline $\begin{array}{l}\text { This has happened more than once:A guy asks me out, often using a } \\
\text { demand for sex instead of 'Hello' to start the conversation. When I } \\
\text { turn him down, he calls me a slut. When I point out the irony of that } \\
\text { bit of name calling, he threatens to rape me. }\end{array}$ & $\begin{array}{l}\text { Threats, Sexual harassment (excluding assault), Denial or trivialization } \\
\text { of sexist misconduct, Slut shaming }\end{array}$ \\
\hline $\begin{array}{l}\text { My female manager only appoints male members of staff to manage a } \\
\text { particular young team because only men would be able to handle the } \\
\text { difficult staff members. }\end{array}$ & $\begin{array}{l}\text { Role stereotyping, Attribute stereotyping, Internalized sexism, Hostile } \\
\text { work environment (excluding pay gap) }\end{array}$ \\
\hline
\end{tabular}

two sets. The higher coverage values seen for our approach could partly account for its state-of-the-art performance.

Table 7 shows few examples of the pseudo-labeled accounts of sexism that are added by our best data augmentation method (Diversity.label $\cap$ support.weakest) using PA as the base classifier. It also shows the categories associated with each post predicted by our PA. It is inferred from these samples that our proposed method is able to predict the correct categories.

For each method, all the hyper-parameter tuning is done using the validation data. The hyper-parameters values for our proposed semi-supervised approaches are as follows. $P_{\text {min }}$ and $P_{\text {max }}$ are set to 4 and 7, respectively. The minimum labeled data coverage $\left(S_{m}\right)$ is set to 1300 by observing the class distribution in the original labeled data. For each method, we pick the optimal confidence threshold $T$ and $T_{o p}$ based on $F_{\text {macro }}$ on the validation set. For Diversity.label $\cap$ support.weakest, our best-performing method, these values are 0.75 and 0.9 , respectively.

The amount of pseudo-labeled data chosen to be added by each of our methods varies according to hyper-parameter values where the best performance on the validation set is observed. For the best augmentation method (Diversity.label $\cap$ support.weakest) using our best classifier (PA) as a base classifier, the highest $F_{\text {macro }}$ is seen at the third iteration, and the corresponding data generated cumulatively till that point amounts to 8994 samples, resulting in the augmented dataset consisting of 22017 labeled samples. The added data sizes for the other data augmentation methods using PA as a base classifier with comparable hyper-parameter configuration range from $8 \mathrm{k}$ to $10 \mathrm{k}$. Our best-proposed approach produces fewer data compared to some other proposed data augmentation methods, confirming the importance of keeping the quality of the pseudo-labeled set high through effective sample selection methods and other mechanisms.

\section{Conclusion}

We investigated semi-supervised learning for the finegrained classification of accounts of sexism using 23 categories of sexism. We proposed a set of methods based on self-training, designed for the multi-label formulation, for capitalizing on unlabeled instances of sexism to augment the training data. We devised a loss function which capitalizes on label confidence scores computed for each pseudolabeled sample in the augmented data. We also proposed an neural architecture involving a domain-adapted BERT model that is trained end-to-end to improve the fine-grained sexism classification performance. We also devised a coarse-to-fine training approach for multi-label sexism classification where we train the models sequentially using categories of sexism of different levels of granularity.

Our proposed methods perform superiorly to a variety of traditional machine learning and deep learning baselines across many standard metrics including Subset Accuracy, the strictest metric. Several of the proposed semi-supervised methods that augment the labeled data with pseudo-labeled samples picked from unlabeled data yield better results than the best baseline (Opti-DL). Our best-performing data augmentation method (Diversity.label $\cap$ Support.weakest) seeks to enhance textual diversity and improve class imbalance. Our proposed sexism classification architecture, which combines biLSTM and attention with a domain-adapted BERT model in an end-to-end trainable manner, also outperforms all baselines. Our best combined method (multi-level PAaug with CBCE loss) further improves the performance; it achieves an instance-based $F$-score $\left(F_{\text {ins }}\right)$ of $0.757, F_{\text {mac }}$ of 0.583 and $S A$ of 0.357 , whereas the best baseline produces an instance-based $F$-score $\left(F_{\text {ins }}\right)$ of $0.714, F_{\text {mac }}$ of 0.546 and $S A$ of 0.242 .

A direction for future work is to tailor and extend the approaches for sexism classification for the identification of sexism, with a view to developing a pipeline in which sexism detection is first carried out to identify posts related to sexism and sexism classification is performed only on 
those posts. Another possible direction is to investigate neural approaches for the identification and categorization of specific forms of sexism such as sexist stereotyping (including but not limited to role stereotyping), sexual harassment and misogyny. Given the presence of social media data in languages other than English, we could also explore multilingual sexism detection and classification.

Open Access This article is licensed under a Creative Commons Attribution 4.0 International License, which permits use, sharing, adaptation, distribution and reproduction in any medium or format, as long as you give appropriate credit to the original author(s) and the source, provide a link to the Creative Commons licence, and indicate if changes were made. The images or other third party material in this article are included in the article's Creative Commons licence, unless indicated otherwise in a credit line to the material. If material is not included in the article's Creative Commons licence and your intended use is not permitted by statutory regulation or exceeds the permitted use, you will need to obtain permission directly from the copyright holder. To view a copy of this licence, visit http://creativecommons.org/licenses/by/4.0/.

\section{References}

1. Abburi H, Parikh P, Chhaya N, Varma V (2020) Semi-supervised multi-task learning for multi-label fine-grained sexism classification. In: Proceedings of the 28th international conference on computational linguistics, international committee on computational linguistics, Barcelona, Spain (Online), pp 5810-5820

2. Abney S (2007) Semisupervised learning for computational linguistics. Chapman and Hall/CRC

3. Agrawal S, Awekar A (2018) Deep learning for detecting cyberbullying across multiple social media platforms. In: European Conference on Information Retrieval, Springer, pp 141-153

4. Anzovino M, Fersini E, Rosso P (2018) Automatic identification and classification of misogynistic language on twitter. In: International conference on applications of natural language to information systems, Springer, pp 57-64

5. Badjatiya P, Gupta S, Gupta M, Varma V (2017) Deep learning for hate speech detection in tweets. In: Proceedings of the 26th international conference on world wide web companion, International World Wide Web Conferences Steering Committee, pp 759-760

6. Burnap P, Williams ML (2016) Us and them: identifying cyber hate on twitter across multiple protected characteristics. EPJ Data Sci 5(1):11

7. Cer D, Yang Y, Kong Sy, Hua N, Limtiaco N, John RS, Constant N, Guajardo-Cespedes M, Yuan S, Tar C, et al. (2018) Universal sentence encoder. arXiv preprint arXiv: 180311175

8. Chiril P, Moriceau V, Benamara F, Mari A, Origgi G, CoulombGully M (2020) An annotated corpus for sexism detection in french tweets. In: Proceedings of the 12th language resources and evaluation conference, pp 1397-1403

9. Chiril P, Moriceau V, Benamara F, Mari A, Origgi G, CoulombGully M (2020) He said “who's gonna take care of your children when you are at acl?': Reported sexist acts are not sexist. In: Proceedings of the 58th Annual Meeting of the Association for Computational Linguistics, pp 4055-4066

10. Chowdhury AG, Sawhney R, Shah R, Mahata D (2019) \# youtoo? detection of personal recollections of sexual harassment on social media. In: Proceedings of the 57th annual meeting of the association for computational linguistics, pp 2527-2537
11. Corazza M, Menini S, Cabrio E, Tonelli S, Villata S (2020) A multilingual evaluation for online hate speech detection. ACM Trans Internet Technol TOIT 20(2):1-22

12. Davidson T, Warmsley D, Macy M, Weber I (2017) Automated hate speech detection and the problem of offensive language. In: Eleventh international aaai conference on web and social media

13. Devlin J, Chang MW, Lee K, Toutanova K (2018) Bert: Pre-training of deep bidirectional transformers for language understanding. arXiv preprint arXiv: 181004805

14. Dutta D, Sircar O (2013) India's winter of discontent: Some feminist dilemmas in the wake of a rape. Fem Stud 39(1):293-306

15. Eccles JS, Jacobs JE, Harold RD (1990) Gender role stereotypes, expectancy effects, and parents' socialization of gender differences. J Soc Issues 46(2):183-201

16. ElSherief M, Belding E, Nguyen D (2017) \# notokay: Understanding gender-based violence in social media. In: Eleventh international AAAI conference on web and social media

17. Frenda S, Ghanem B, Montes-y Gómez M, Rosso P (2019) Online hate speech against women: automatic identification of misogyny and sexism on twitter. J Intell Fuzzy Syst 36(5):4743-4752

18. Gao L, Kuppersmith A, Huang R (2017) Recognizing explicit and implicit hate speech using a weakly supervised two-path bootstrapping approach. In: Proceedings of the Eighth International Joint Conference on Natural Language Processing (Volume 1: Long Papers), pp 774-782

19. Jafarpour B, Matwin S et al. (2018) Boosting text classification performance on sexist tweets by text augmentation and text generation using a combination of knowledge graphs. In: Proceedings of the 2 nd workshop on abusive language online (ALW2), pp $107-114$

20. Jha A, Mamidi R (2017) When does a compliment become sexist? analysis and classification of ambivalent sexism using twitter data. In: Proceedings of the second workshop on NLP and computational social science, pp 7-16

21. Karlekar S, Bansal M (2018) Safecity: understanding diverse forms of sexual harassment personal stories. In: Proceedings of the 2017 conference on empirical methods in natural language processing (EMNLP), pp 2805-2811

22. Khatua A, Cambria E, Khatua A (2018) Sounds of silence breakers: exploring sexual violence on twitter. In: 2018 IEEE/ACM international conference on advances in social networks analysis and mining (ASONAM), pp 397-400

23. Kim Y (2014) Convolutional neural networks for sentence classification. In: Proceedings of the 2017 conference on empirical methods in natural language processing (EMNLP), pp 1746-1751

24. Mead M (1963) Sex and temperament in three primitive societies, vol 370. Morrow New York

25. Melville S, Eccles K, Yasseri T (2018) Topic modelling of everyday sexism project entries. Front Dig Human 5:28

26. Menon N (2012) Seeing like a feminist. Penguin, Westminster

27. Nobata C, Tetreault J, Thomas A, Mehdad Y, Chang Y (2016) Abusive language detection in online user content. In: Proceedings of the 25th international conference on world wide web, International World Wide web conferences steering committee, pp 145-153

28. Parikh P, Abburi H, Badjatiya P, Krishnan R, Chhaya N, Gupta M, Varma V (2019) Multi-label categorization of accounts of sexism using a neural framework. In: Proceedings of the 2019 conference on empirical methods in natural language processing and the 9th international joint conference on natural language processing (EMNLP-IJCNLP), pp 1642-1652

29. Pennebaker JW, Boyd RL, Jordan K, Blackburn K (2015) The development and psychometric properties of liwc2015. Tech. rep

30. Pennington J, Socher R, Manning C (2014) Glove: Global vectors for word representation. In: Proceedings of the 2014 conference 
on empirical methods in natural language processing (EMNLP), pp 1532-1543

31. Peters ME, Neumann M, Iyyer M, Gardner M, Clark C, Lee K, Zettlemoyer L (2018) Deep contextualized word representations. In: Proceedings of NAACL

32. Plaza-Del-Arco FM, Molina-González MD, Ureña-López LA, Martín-Valdivia MT (2020) Detecting misogyny and xenophobia in spanish tweets using language technologies. ACM Trans Internet Technol TOIT 20(2):1-19

33. Qian J, ElSherief M, Belding E, Wang WY (2018) Hierarchical cvae for fine-grained hate speech classification. In: Proceedings of the 2018 conference on empirical methods in natural language processing, pp 3550-3559

34. Rodríguez-Sánchez F, Carrillo-de Albornoz J, Plaza L (2020) Automatic classification of sexism in social networks: an empirical study on twitter data. IEEE Access 8:219563-219576

35. Schrading N, Alm CO, Ptucha R, Homan C (2015) An analysis of domestic abuse discourse on reddit. In: Proceedings of the 2015 conference on empirical methods in natural language processing, pp 2577-2583

36. Suvarna A, Bhalla G (2020) \# notawhore! a computational linguistic perspective of rape culture and victimization on social media. In: Proceedings of the 58th annual meeting of the association for computational linguistics: student research workshop, pp 328-335

37. Van Hee C, Lefever E, Verhoeven B, Mennes J, Desmet B, De Pauw G, Daelemans W, Hoste V (2015) Detection and finegrained classification of cyberbullying events. In: Proceedings of the international conference recent advances in natural language processing, pp 672-680

38. Wang J, Yu LC, Lai KR, Zhang X (2016) Dimensional sentiment analysis using a regional cnn-lstm model. In: Proceedings of the 54th Annual Meeting of the Association for Computational Linguistics (Volume 2: Short Papers), vol 2, pp 225-230
39. Warner W, Hirschberg J (2012) Detecting hate speech on the world wide web. In: Proceedings of the second workshop on language in social media, Association for Computational Linguistics, pp 19-26

40. Waseem Z, Hovy D (2016) Hateful symbols or hateful people? predictive features for hate speech detection on twitter. In: Proceedings of the NAACL student research workshop, pp 88-93

41. Xiao H (2018) bert-as-service. https://github.com/hanxiao/bertas-service

42. Yan P, Li L, Chen W, Zeng D (2019) Quantum-inspired density matrix encoder for sexual harassment personal stories classification. In: 2019 IEEE international conference on intelligence and security informatics (ISI), IEEE, pp 218-220

43. Yang Z, Yang D, Dyer C, He X, Smola A, Hovy E (2016) Hierarchical attention networks for document classification. In: Proceedings of the 2016 conference of the North American chapter of the association for computational linguistics: human language technologies, pp 1480-1489

44. Yarowsky D (1995) Unsupervised word sense disambiguation rivaling supervised methods. In: 33rd annual meeting of the association for computational linguistics, pp 189-196

45. Zhang ML, Zhou ZH (2014) A review on multi-label learning algorithms. IEEE Trans Knowl Data Eng 26(8):1819-1837

46. Zhang Z, Luo L (2018) Hate speech detection: A solved problem? the challenging case of long tail on twitter. Semantic Web pp 1-21

47. Zhong H, Li H, Squicciarini AC, Rajtmajer SM, Griffin C, Miller DJ, Caragea C (2016) Content-driven detection of cyberbullying on the instagram social network. In: IJCAI, pp 3952-3958

48. Zhou C, Sun C, Liu Z, Lau F (2015) A c-lstm neural network for text classification. arXiv preprint arXiv: 151108630 